\title{
A mathematical model of anaerobic digestion with syntrophic relationship, substrate inhibition and distinct removal rates *
}

\author{
Radhouane Fekih-Salem ${ }^{\ddagger \dagger}$, Yessmine Daoud ${ }^{\ddagger}$, Nahla Abdellatif ${ }^{\S}$, and Tewfik Sari ${ }^{\uparrow}$
}

\begin{abstract}
Understanding and exploiting the syntrophic relationship between microbial species is a major challenge in the mathematical theory of the anaerobic digestion process. In this work, we focus on the acetogenesis and hydrogenotrophic methanogenesis phases and we include distinct removal rates for the species. Our study gives a quite comprehensive analysis of a syntrophic model by analyzing the joined effects of syntrophy relationship, mortality, substrate inhibition and input concentrations that were neglected in previous studies. The mathematical analysis of the model involving the mortality is a difficult problem since the model is not reduced to a planar system as in the case where the dilution rates of the substrates and the removal rates of microbial species are equal. Using general nonmonotonic growth rates, the necessary and sufficient conditions of existence and local stability of all steady states of the four-dimensional system are determined, according to the operating parameters. This general model exhibits a rich behavior with the coexistence of two microbial species, the bistability, the multiplicity of coexistence steady states, and the existence of two steady states of extinction of the first species. The operating diagram shows how the model behaves by varying the control parameters and illustrates the effect of the substrate inhibition and the new input substrate concentration (hydrogen) on the appearance or the disappearance of coexistence and bistability regions. Similarly to the classical chemostat model, including the substrate inhibition can destabilize a two-tiered microbial 'food chain', where the asymptotic behavior of the system depends on the initial condition.
\end{abstract}

Key words. Anaerobic digestion, chemostat, syntrophy, inhibition, bistability, operating diagram

AMS subject classifications. 34A34, 34D20, 37N25, 92B05

1. Introduction. Anaerobic Digestion (AD) is a process used for the biological treatment of municipal, agricultural and industrial wastes with the additional benefit of producing energy in the form of biogas. During this process, the waste is first partially transformed into volatile fatty acids and then converted into methane and carbon dioxide, which can be used as a carbon source for microalgae [30]. $\mathrm{AD}$ process is too complex with difficulty to collect informative experimental data which complicates the model validation and the parameter identification [15]. The generic AD Model No.1 (ADM1) of the IWA Task Group for Mathematical Modeling of AD Processes is characterized by its extreme complexity with 32 dynamic concentration state variables and a large number of parameters [3].

Many mathematical models describing the whole process or some key steps have been considered in the last three decades; see [5, 7, 10, 17, 21, 25, 40, 41, 42, 49, 51, 52, 53]. A synthetic and unified vision of many models involving two or three cross-feeding species and various types of inhibition has been proposed in [14]. Using specific growth functions, the numerical simulations reveal the reduction in both productivity and stability due to inhibitions with the occurrence of stable periodic orbits owing to the presence of negative and positive feedback loops. In [25], a mathematical analysis of the protein-rich Microalgae AD model (the so-called MAD) shows the process behavior according to the control parameters where the operating diagram illustrates the ideal conditions to optimize biogas yield and ammonia toxicity. In fact, the MAD model has been proposed in [28] and was validated from experimental data of an AD process of Chlorella vulgaris microalgae involving four substrates and three microbial species with three reactions and two steps (hydrolysis-acetogenesis and methanogenesis).

Recently, a complete mathematical analysis was provided in [31] of a two-step model (acidogenesis and methanogenesis) introduced in [9] where a fifth state variable (ammonia) is included. The decay and the inhibition caused by ammonia were taken into account by considering a general class of response functions. In $[52,53]$, an eight-dimensional mathematical model describing three of the

\footnotetext{
*Submitted to the editors 2020-10-27.

Funding: This work was supported by the Euro-Mediterranean research network TREASURE (http://www.inra.fr/ treasure).

${ }^{\dagger}$ University of Monastir, Higher Institute of Computer Science of Mahdia, Tunisia (radhouene.fekihsalem@isima.rnu.tn).

${ }^{\ddagger}$ University of Tunis El Manar, National Engineering School of Tunis, LAMSIN, Tunisia (yessmine.daoud@enit.utm.tn).

$\S$ University of Manouba, National School of Computer Science, Tunisia (nahla.abdellatif@ensi-uma.tn).

ॠITAP, Univ Montpellier, INRAE, Institut Agro, Montpellier, France (tewfik.sari@inrae.fr).
} 
four main stages of $\mathrm{AD}$ (acidogenesis, acetogenesis, and methanogenesis) was analyzed by considering syntrophy and substrate inhibition effects. Following [51] and using general functional responses, a three-tiered microbial food-web model was studied in [41] discovering the emergence of the coexistence region in the operating diagram where a stable limit cycle is born via the Hopf bifurcation, which has not been reported by [51]. The work of [41] has been recently extended in [34, 35] by considering the effects of the phenol and hydrogen input concentrations, together with the effects of maintenance (or decay) terms.

Using a step by step parameter identification procedure, Bernard et al. [7] have proposed and have validated a reduced two-step model (the so-called AM2) from experimental data of the AD process. This model has a cascade structure and has been widely applied for control and optimization of AD process [21, 42, 43, 44]. Using a maximum likelihood principal component analysis [27] and generated data built from ADM1 model, the appropriate number of reactions is determined by a systematic data driven-approach followed by a parameter identification procedure [22]. The resulting low-order model is the two-tiered microbial 'food chain' leading to perfectible direct and cross-validation results. The AM2 model was mathematically studied in $[5,38]$ and was extended in $[4,6]$, where a fifth state variable (SMP: Soluble Microbial Products), important for fouling of membranes, is included. For a review of mathematical modeling of anaerobic digestion with respect to theory, applications and technologies, the reader is refereed to [50].

The two-tiered microbial model we consider here describes the next two biological reactions:

$$
s_{0} \stackrel{\mu_{0}}{\longrightarrow} x_{0}+s_{1}, \quad s_{1} \stackrel{\mu_{1}}{\longrightarrow} x_{1}
$$

where a substrate $s_{0}$ (Volatile Fatty Acid) is consumed by a biomass $x_{0}$ (acetogenic bacteria) to produce a product $s_{1}$ (hydrogen). The substrate $s_{1}$ is consumed in the second reaction by another biomass $x_{1}$ (hydrogenotrophic methanogenic bacteria). $\mu_{0}$ and $\mu_{1}$ are the bacterial growth rates, depending eventually on one or both substrates. The substrates $s_{0}$ and $s_{1}$ are introduced in the reactor with the inflowing concentrations $s_{0}^{i n}$ and $s_{1}^{i n}$, respectively, and a dilution rate $D$. These reactions are described by the following system of differential equations

$$
\left\{\begin{array}{l}
\dot{s}_{0}=D\left(s_{0}^{\text {in }}-s_{0}\right)-\mu_{0}(\cdot) x_{0}, \\
\dot{x}_{0}=\left(\mu_{0}(\cdot)-D_{0}\right) x_{0}, \\
\dot{s}_{1}=D\left(s_{1}^{\text {in }}-s_{1}\right)+\mu_{0}(\cdot) x_{0}-\mu_{1}(\cdot) x_{1}, \\
\dot{x}_{1}=\left(\mu_{1}(\cdot)-D_{1}\right) x_{1},
\end{array}\right.
$$

where $D_{0}$ and $D_{1}$ represent, respectively, the disappearance rates of acetogenic and methanogenic bacteria. In this study, the two-tiered model (1.2) is analyzed where $D_{i}$ can be modeled as in $[29,45]$ by

$$
D_{i}=\alpha_{i} D+a_{i}, \quad i=0,1,
$$

where the nonnegative death (or decay) rate parameters $a_{0}$ and $a_{1}$ are taken into consideration with units of the dilution rate ( $D$ has units $1 / \mathrm{d})$. These decay terms included in model (1.2) are related to consumption of energy, other than growth; see for instance [23] or [33]. The coefficients $\alpha_{0}$ and $\alpha_{1}$ belong to $[0,1]$ and represent, respectively, the first and the second biomass proportion that leaves the reactor. For example, in [7] these coefficients are proposed to model a biomass reactor attached to the support or to decouple the residence time of solids and the hydraulic residence time $(1 / D)$. Thus, the study will not be restricted to the case $\alpha_{i}=1, i=0,1$, as in most of the studies in the literature (see Tables 1 and 2 below), and the case $0 \leq \alpha_{i} \leq 1$, which is of biological interest, will be investigated.

If the growth rate $\mu_{0}$ depends only on substrate $s_{0}$ and $\mu_{1}$ depends only on $s_{1}$, that is,

$$
\mu_{0}(\cdot)=\mu_{0}\left(s_{0}\right), \quad \mu_{1}(\cdot)=\mu_{1}\left(s_{1}\right),
$$

then system (1.2) has a cascade structure and describes a commensalistic relationship where the commensal species $x_{1}$ needs the first species $x_{0}$ to grow, while $x_{0}$ can grow without $x_{1}$ and it is not 
affected by the growth of the commensal species $x_{1}$. If $\mu_{0}$ depends on both substrates $s_{0}$ and $s_{1}$, and $\mu_{1}$ depends on substrate $s_{1}$, that is,

$$
\mu_{0}(\cdot)=\mu_{0}\left(s_{0}, s_{1}\right), \quad \mu_{1}(\cdot)=\mu_{1}\left(s_{1}\right),
$$

then system (1.2) describes a syntrophic relationship where two microbial species depend on each other for survival by the production of a required substrate $s_{1}$. In this case, each species benefits from the presence of the other species. Tables 1 and 2 summarize the modeling assumptions made in the literature on two-tiered model (1.2) describing the commensalistic and the syntrophic relationships, respectively, according to the input concentration $s_{1}^{\text {in }}$, the removal rates $D_{i}$, and the choice of the growth functions.

Table 1

Literature examples of the commensalistic relationship of two-tiered model (1.2), the modeling assumptions and the description of the growth rates (1.4).

\begin{tabular}{lllll} 
References & $s_{1}^{\text {in }}$ & $D_{i}$ & $\mu_{0}\left(s_{0}\right)$ & $\mu_{1}\left(s_{1}\right)$ \\
\hline Reilly [37], & 0 & $D$ & Monod & Monod \\
Simeonov and Stoyanov [47] & 0 & $D+a_{i}$ & Monod & Monod \\
Stephanopoulos [48] & 0 & $D$ & Monotonic & Monotonic or Nonmonotonic \\
Bernard et al. [7] & $\geq 0$ & $\alpha D$ & Monod & Haldane \\
Simeonov and Diop [46] & 0 & $D$ & Monod or Contois & Haldane \\
Sbarciog et al. [42] & $\geq 0$ & $D$ & Monotonic & Nonmonotonic \\
Benyahia et al. [5] & $\geq 0$ & $\alpha D$ & Monotonic & Nonmonotonic
\end{tabular}

Table 2

Literature examples of the syntrophic relationship of two-tiered model (1.2), the modeling assumptions and the description of the growth rates (1.5).

\begin{tabular}{lllll} 
References & $s_{1}^{i n}$ & $D_{i}$ & $\mu_{0}\left(s_{0}, s_{1}\right)$ & $\mu_{1}\left(s_{1}\right)$ or $\mu_{1}\left(s_{0}, s_{1}\right)$ \\
\hline Kreikenbohm and Bohl [26] & 0 & $D$ & Monod in $s_{0}$, decreasing in $s_{1}$ & Monod \\
Burchard [10], El-Hajji et al. [16] & 0 & $D$ & Increasing in $s_{0}$, decreasing in $s_{1}$ & Increasing \\
Xu et al. [54] & 0 & $D+a_{i}$ & Increasing in $s_{0}$, decreasing in $s_{1}$ & Monod \\
Sari et al. [39] & $\geq 0$ & $D$ & Increasing in $s_{0}$, decreasing in $s_{1}$ & Decreasing in $s_{0}$ increasing in $s_{1}$ \\
Harvey et al. [24] & 0 & $D$ & Increasing in $s_{0}$, decreasing in $s_{1}$ & Nonmonotonic \\
Sari and Harmand [40] & 0 & $D+a_{i}$ & Increasing in $s_{0}$, decreasing in $s_{1}$ & Increasing \\
Fekih et al. [18] & 0 & $D+a_{i}$ & Increasing in $s_{0}$, decreasing in $s_{1}$ & Nonmonotonic \\
Daoud et al. [11] & $\geq 0$ & $D+a_{i}$ & Increasing in $s_{0}$, decreasing in $s_{1}$ & Increasing
\end{tabular}

Harvey et al. [24] have studied model (1.2) in the particular case where $s_{1}^{i n}=0, D_{i}=D$, and the growth rate $\mu_{0}\left(s_{0}, s_{1}\right)=f\left(s_{0}\right) \cdot g\left(s_{1}\right)$ with $f$ is increasing in $s_{0}$ and $g$ is decreasing in $s_{1}$. Our study provides an extension of the results in [24] to the case where $D_{1}$ and $D_{2}$ are distinct from $D$. Notice that most of the studies in the existing literature (see Table 2) consider the case of equal removal rates $\left(D_{1}=D_{2}=D\right)$, where the model can be reduced to a two-dimensional system. In this paper, we generalize $[10,16,24,26]$, by allowing distinct removal rates. In this case, the study of the stability is much more delicate and requires the Liénard-Chipart stability criteria [20] for a four-dimensional system. Furthermore, it is reported in the literature [5, 7, 18, 24, 42, 46, 48] that at many times the second reaction of (1.1) is inhibited by large values of $s_{1}$, which instigates to consider a Haldane-type growth function for $\mu_{1}$. The goal of the present work is to understand the joined effects of syntrophy, mortality of two microbial species, substrate inhibition on their growth and inflowing substrate concentration of the second species, which have not been studied in the literature. Moreover, here, we do not specify kinetics but we consider qualitative properties on the growth functions and we assume that the second species is inhibited when the concentration of substrate becomes significant. The particular case $s_{1}^{i n}=0$ was considered in [18]. The case where $\mu_{1}$ does not present inhibition was considered in [11, 40, 54].

On the other hand, our study provides an important tool for the experimentation which is the operating diagram showing the behavior of the syntrophic model (1.2) according to the control parameters $D, s_{0}^{i n}$ and $s_{1}^{i n}$, when all biological parameters are fixed. This operating diagram is often studied 
numerically or theoretically both in the biological literature [36, 42, 51, 54] and the mathematical literature $[1,2,11,12,13,18,19,23,25,32,38,40,41,52,53]$.

This paper is organized as follows: in section 2, we present the assumptions made on the growth functions and give some preliminary results. Section 3 is devoted to the analysis of steady states and their local stability. In section 4 , we present the operating diagrams which depict the different outcomes of the model according to control parameters. Finally, some conclusions are drawn in section 5 . The definition domains of some auxiliary functions used for the description of the steady states with their conditions of existence and stability are given in Appendix A. The proofs of all results are reported in Appendix B. With specific growth rates satisfying the general assumptions, the maximal number of solutions of an equation which determines some definition domains are given in Appendix C. Finally, some tables are given in Appendix D.

2. Mathematical model and assumptions. In what follows, we study model (1.2) where the removal rates $D_{i}$ and the growth rates $\mu_{i}, i=0,1$ are given by (1.3) and (1.5), respectively. Thus, the syntrophic model can be written as follows

$$
\left\{\begin{array}{l}
\dot{s}_{0}=D\left(s_{0}^{i n}-s_{0}\right)-\mu_{0}\left(s_{0}, s_{1}\right) x_{0}, \\
\dot{x}_{0}=\left(\mu_{0}\left(s_{0}, s_{1}\right)-D_{0}\right) x_{0}, \\
\dot{s}_{1}=D\left(s_{1}^{i n}-s_{1}\right)+\mu_{0}\left(s_{0}, s_{1}\right) x_{0}-\mu_{1}\left(s_{1}\right) x_{1}, \\
\dot{x}_{1}=\left(\mu_{1}\left(s_{1}\right)-D_{1}\right) x_{1} .
\end{array}\right.
$$

We first make the following general assumptions on the bacterial growth rates. The functions $\mu_{0}$ and $\mu_{1}$ belong to $\mathcal{C}^{1}\left(\mathbb{R}^{+}, \mathbb{R}^{+}\right)$and $\mathcal{C}^{1}\left(\mathbb{R}^{+}\right)$, respectively and verify:

Hypothesis 2.1. Growth of species $x_{0}$ can take place if and only if the substrate $s_{0}$ is present: $\mu_{0}\left(0, s_{1}\right)=0,0<\mu_{0}\left(s_{0}, s_{1}\right)<+\infty$, for all $s_{0}>0$ and $s_{1} \geq 0$.

Hypothesis 2.2. Growth of species $x_{1}$ can take place if and only if the substrate $s_{1}$ is present: $\mu_{1}(0)=0$ and $\mu_{1}\left(s_{1}\right)>0$, for all $s_{1}>0$.

Hypothesis 2.3. Growth rate of species $x_{0}$ is favored by $s_{0}$ and is inhibited by the substrate $s_{1}$ : $\frac{\partial \mu_{0}}{\partial s_{0}}\left(s_{0}, s_{1}\right)>0$ and $\frac{\partial \mu_{0}}{\partial s_{1}}\left(s_{0}, s_{1}\right)<0$, for all $s_{0}>0$ and $s_{1}>0$.

Hypothesis 2.4. The nonmonotonic growth function $\mu_{1}$ takes into account the growth-limiting for low concentrations of substrate $s_{1}$ and the growth-inhibiting for high concentrations: $\mu_{1}\left(s_{1}\right)$ reaches a maximum value $\mu_{1}^{\max }:=\mu_{1}\left(s_{1}^{\max }\right)$ at $s_{1}=s_{1}^{\max }$ and satisfies $\mu_{1}^{\prime}\left(s_{1}\right)>0$, for all $s_{1} \in\left[0, s_{1}^{\max }\right)$, $\mu_{1}^{\prime}\left(s_{1}\right)<0$, for all $\left(s_{1}^{\max },+\infty\right)$ and $\mu_{1}(+\infty)=0$.

Hypothesis 2.5. The maximum growth rate of the species $x_{0}$ decreases with the concentration of substrate $s_{1}$ : for all $s_{1}>0, \bar{\mu}_{0}^{\prime}\left(s_{1}\right)<0$ where $\bar{\mu}_{0}\left(s_{1}\right):=\sup _{s_{0} \geq 0} \mu_{0}\left(s_{0}, s_{1}\right)$.

The following result proves that syntrophic model (2.1) preserves the biological significance where all solutions of the system are nonnegative and bounded for any nonnegative initial condition.

Proposition 2.6. For any nonnegative initial condition, the solution of system (2.1) exists for all nonnegative times, remains nonnegative and is positively bounded. In addition, the set

$$
\Omega=\left\{\left(s_{0}, x_{0}, s_{1}, x_{1}\right) \in \mathbb{R}_{+}^{4}: 2 s_{0}+x_{0}+s_{1}+x_{1} \leq \frac{D}{D_{\min }}\left(2 s_{0}^{i n}+s_{1}^{i n}\right)\right\},
$$

where $D_{\min }=\min \left(D, D_{0}, D_{1}\right)$, is positively invariant and a global attractor for (2.1).

3. Analysis of the syntrophic model. A steady state exists if and only if all its components are nonnegative. Model (2.1) can have at most six steady states, which we denote as follows:

- $\mathrm{SS}_{0}\left(x_{0}=x_{1}=0\right)$ : the washout of both species.

- $\mathrm{SS}_{1}\left(x_{1}=0, x_{0}>0\right)$ : species $x_{1}$ is extinct while species $x_{0}$ survives.

- $\mathrm{SS}_{2}^{i}, i=1,2\left(x_{0}>0, x_{1}>0\right)$ : both species are maintained.

- $\mathrm{SS}_{3}^{i}, i=1,2\left(x_{0}=0, x_{1}>0\right)$ : species $x_{0}$ is extinct while species $x_{1}$ survives.

We show below that all steady states are unique, if they exist. However, bifurcations may occur (see Table 8) where two steady states collide, giving rise to a non hyperbolic steady state. First, we 
introduce in Table 3 the auxiliary functions for determining the existence and stability conditions. Some comments and details on their definition domains are given in Appendix A. In the particular case of specific growth rates of Monod-type with hydrogen inhibition and of Haldane-type (C.1), the auxiliary functions defined in Table 3 can be calculated analytically and are given in Table 12 .

Table 3

Auxiliary functions where $\operatorname{dom}\left(F_{0}\right)$ and $I_{j}, j=1,2$ are given in Table 9.

Definition

\begin{tabular}{ll}
\hline$s_{0}=M_{0}\left(y, s_{1}\right)$ & $\begin{array}{l}\text { Let } s_{1} \geqslant 0 . s_{0}=M_{0}\left(y, s_{1}\right) \text { is the unique solution of equation } y=\mu_{0}\left(s_{0}, s_{1}\right) . \\
\text { It is defined for } y \in\left[0, \bar{\mu}_{0}\left(s_{1}\right)\right) \\
s_{1}=M_{1}^{1}(y) \text { is the unique solution in }\left[0, s_{1}^{\text {max }}\right] \text { of equation } y=\mu_{1}\left(s_{1}\right) . \\
s_{1}=M_{1}^{1}(y)\end{array}$ \\
$\begin{array}{l}\text { It is defined for } y \in\left[0, \mu_{1}^{\text {max }}\right] . \\
s_{1}=M_{1}^{2}(y) \text { is the unique solution in }\left[s_{1}^{\text {max }},+\infty\right) \text { of equation } y=\mu_{1}\left(s_{1}\right) . \\
\text { It is defined for } y \in\left(0, \mu_{1}^{\text {max }}\right] .\end{array}$ \\
\hline$F_{0}\left(D, s_{1}^{i n}\right)$ & $F_{0}\left(D, s_{1}^{\text {in }}\right)=M_{0}\left(\alpha_{0} D+a_{0}, s_{1}^{\text {in }}\right)$ defined for $\left(D, s_{1}^{\text {in }}\right) \in \operatorname{dom}\left(F_{0}\right)$ \\
$F_{1}^{j}(D)$ & $F_{1}^{j}(D)=M_{0}\left(\alpha_{0} D+a_{0}, M_{1}^{j}\left(\alpha_{1} D+a_{1}\right)\right), D \in I_{j}$ \\
$F_{2}^{j}(D)$ & $F_{2}^{j}(D)=M_{1}^{j}\left(\alpha_{1} D+a_{1}\right)+F_{1}^{j}(D), D \in I_{j}$
\end{tabular}

The following result gives all the steady states of (2.1) and the necessary and sufficient conditions of their existence and stability. For convenience, we shall use the abbreviation LES for Locally Exponentially Stable.

Proposition 3.1. Assume that Hypotheses 2.1 to 2.4 hold. Then, the six steady states of (2.1) are given in Table 4. The conditions of their existence and stability are given in Table 5.

Table 4

Steady states of (2.1). All functions are defined in Table 3.

\begin{tabular}{lll} 
& $s_{0}, s_{1}$ components & $x_{0}, x_{1}$ components \\
\hline $\mathrm{SS}_{0}$ & $s_{0}=s_{0}^{i n}, s_{1}=s_{1}^{i n}$ & $x_{0}=0, x_{1}=0$ \\
\hline \multirow{2}{*}{$\mathrm{SS}_{1}$} & $s_{0}$ is a solution of equation & $x_{0}=\frac{D}{D_{0}}\left(s_{0}^{i n}-s_{0}\right)$ \\
& $\mu_{0}\left(s_{0}, s_{0}^{i n}+s_{1}^{i n}-s_{0}\right)=D_{0}$ & $x_{1}=0$ \\
& $s_{1}=s_{0}^{i n}+s_{1}^{i n}-s_{0}$ & $x_{0}=\frac{D}{D_{0}}\left(s_{0}^{i n}-s_{0}\right)$ \\
\hline \multirow{2}{*}{$\mathrm{SS}_{2}^{j}$} & $s_{0}=F_{1}^{j}(D)$ & $x_{1}=\frac{D}{D_{1}}\left(s_{0}^{i n}+s_{1}^{i n}-s_{0}-s_{1}\right)$ \\
\hline \multirow{2}{*}{$\mathrm{SS}_{3}^{j}$} & $s_{1}=M_{1}^{j}\left(D_{1}\right)$ & $x_{0}=0$ \\
& $s_{1}=s_{0}^{i n}$ & $x_{1}=\frac{D}{D_{1}}\left(s_{1}^{i n}-M_{1}^{j}\left(D_{1}\right)\right)$
\end{tabular}

Table 5

Necessary and sufficient conditions of existence and local stability of steady states of model (2.1).

Existence condition

\begin{tabular}{ll}
\hline $\mathrm{SS}_{0}$ & always exists \\
$\mathrm{SS}_{1}$ & $s_{0}^{i n}>F_{0}\left(D, s_{1}^{i n}\right)$ \\
$\mathrm{SS}_{2}^{1}$ & $s_{0}^{i n}>\max \left(F_{1}^{1}(D), F_{2}^{1}(D)-s_{1}^{i n}\right)$ \\
$\mathrm{SS}_{2}^{2}$ & $s_{0}^{i n}>\max \left(F_{1}^{2}(D), F_{2}^{2}(D)-s_{1}^{i n}\right)$ \\
$\mathrm{SS}_{3}^{1}$ & $s_{1}^{\text {in }}>M_{1}^{1}\left(D_{1}\right)$, \\
$\mathrm{SS}_{3}^{2}$ & $s_{1}^{\text {in }}>M_{1}^{2}\left(D_{1}\right)$,
\end{tabular}

Stability condition

$s_{0}^{\text {in }}<F_{0}\left(D, s_{1}^{\text {in }}\right)$ and $\left(s_{1}^{\text {in }}<M_{1}^{1}\left(D_{1}\right)\right.$ or $\left.s_{1}^{\text {in }}>M_{1}^{2}\left(D_{1}\right)\right)$

$s_{0}^{i n}+s_{1}^{i n}<F_{2}^{1}(D)$ or $s_{0}^{i n}+s_{1}^{i n}>F_{2}^{2}(D)$

LES whenever it exists

Always unstable

$s_{0}^{\text {in }}<F_{1}^{1}(D)$

Always unstable

Remark 3.2. Since the function $F_{0}\left(D, s_{1}^{i n}\right)$ is defined for $\left(D, s_{1}^{i n}\right) \in \operatorname{dom}\left(F_{0}\right)$ (see Proposition A.1), the condition $s_{0}^{\text {in }}>F_{0}\left(D, s_{1}^{\text {in }}\right)$ means that $\left(D, s_{1}^{i n}\right) \in \operatorname{dom}\left(F_{0}\right)$ and the inequality is satisfied. Conversely, if $\left(D, s_{1}^{\text {in }}\right) \notin \operatorname{dom}\left(F_{0}\right)$, we let $F_{0}\left(D, s_{1}^{i n}\right)=+\infty$. Thus, the condition $s_{0}^{\text {in }}<F_{0}\left(D, s_{1}^{i n}\right)$ means that $\left(D, s_{1}^{i n}\right) \notin \operatorname{dom}\left(F_{0}\right)$ or the inequality is satisfied and $\left(D, s_{1}^{i n}\right) \in \operatorname{dom}\left(F_{0}\right)$. Similarly, the condition $s_{1}^{\text {in }}>M_{1}^{j}\left(D_{1}\right)$ means that $D \in \bar{I}_{j}, j=1,2$ and the inequality is satisfied, while the condition $s_{1}^{i n}<M_{1}^{j}\left(D_{1}\right)$ means $D \notin \bar{I}_{j}$ or the inequality is satisfied and $D \in \bar{I}_{j}$. The other conditions involving functions $F_{i}^{j}(D), i, j=1,2$, follow similarly.

As we will see in Proposition 4.5, the limit case $D=\bar{D}_{1}$ corresponds to saddle-node bifurcations of $\mathrm{SS}_{2}^{1}$ with $\mathrm{SS}_{2}^{2}$ and $\mathrm{SS}_{3}^{1}$ with $\mathrm{SS}_{3}^{2}$ where these steady states are non hyperbolic. In the particular case 
$s_{1}^{i n}=0$, we obtain the same result as in $[18,40]$ where $\mathrm{SS}_{3}^{1}$ and $\mathrm{SS}_{3}^{2}$ do not exist since the conditions of their existence in Table 5 are not satisfied. Compared to [40], a main change in the existence of steady states of our model (2.1) is the appearance of the second positive steady state $\mathrm{SS}_{2}^{2}$ and two steady states $\mathrm{SS}_{3}^{1}$ and $\mathrm{SS}_{3}^{2}$.

4. Operating diagrams. The operating diagram is a very useful tool to determine the asymptotic behavior of the process with respect to the control parameters $D, s_{0}^{i n}$ and $s_{1}^{i n}$ which are the most easily parameters to manipulate in a chemostat. All other parameters are fixed since they have biological meaning and cannot be easily manipulated by the biologist. The biological parameter values used in all figures are provided in Table 13. To construct the operating diagram, we first define in Table 6 the set of surfaces $\Gamma=\left\{\gamma_{0}, \gamma_{i}^{j}, \gamma_{4}, i=1,2,3, j=1,2\right\}$ which are the boundaries of different regions of the $\left(D, s_{0}^{i n}, s_{1}^{i n}\right)$-space. We define also in Table 6 the curve $\mathcal{C}_{j}$ of the function $y=M_{1}^{j}\left(D_{1}\right)-s_{1}^{i n}, j=1,2$ to determine its sign according to $s_{1}^{i n}$ and $D$. As we will see in Propositions 4.2 to 4.4 , if $D$ is fixed in $\bar{I}_{j}$, then $s_{1}^{i n}=s_{1 j}^{i n *}=M_{1}^{j}\left(\alpha_{1} D+a_{1}\right)$ and if $s_{1}^{i n}$ is fixed, the equation $M_{1}^{j}\left(\alpha_{1} D+a_{1}\right)=s_{1}^{i n}$ can have a unique solution $D=D_{j}^{*}$ with $j=1,2$. As stated in the following result, the surfaces in the set $\Gamma$ separate the operating space $\left(D, s_{0}^{i n}, s_{1}^{\text {in }}\right)$ into twelve regions, denoted $\mathcal{J}_{k}, k=1, \ldots, 12$, and defined in Table 7.

Table 6

The set of surfaces $\Gamma$, the curves $\mathcal{C}_{1}$ and $\mathcal{C}_{2}$, and the corresponding colors in Figures 1 and 3 to 6.

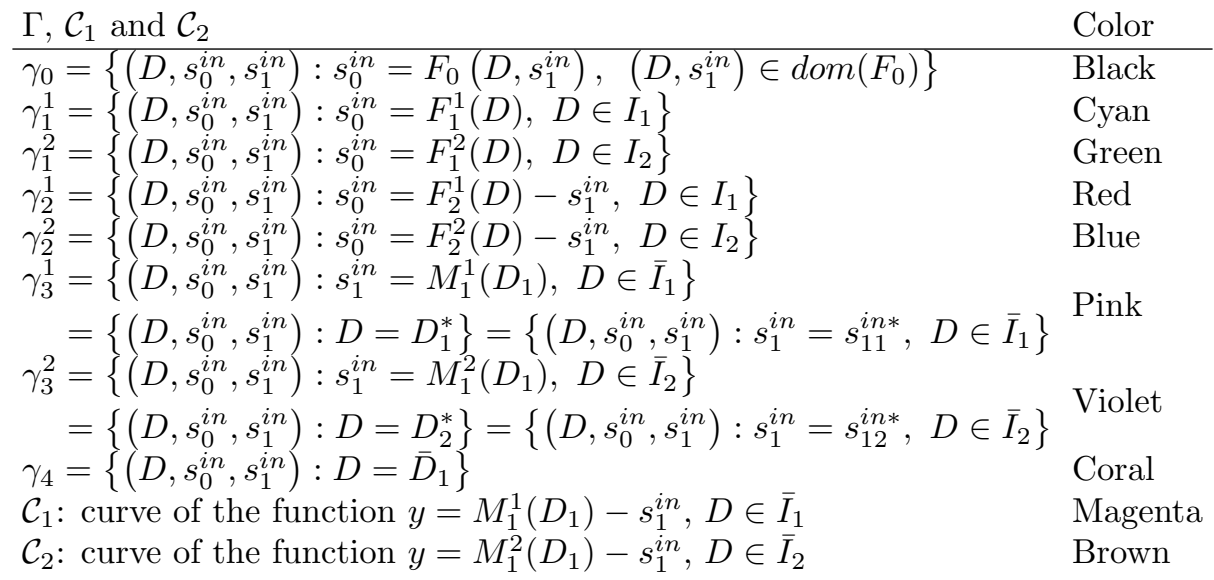

Proposition 4.1. Assume that Hypotheses 2.1 to 2.5 hold. The existence and the stability of the steady states of (2.1) in the twelve regions $\mathcal{J}_{k}, k=1, \ldots, 12$ of the operating diagram are determined in Table 7.

Table 7

Existence and stability of steady states in the regions of the operating diagram. The letter $S$ (resp. U) means that the corresponding steady state is LES (resp. unstable). No letter means that the steady state does not exist.

\begin{tabular}{|c|c|c|c|c|c|c|c|c|c|}
\hline Condition 1 & Condition 2 & Region & Color & $\mathrm{SS}_{0}$ & $\mathrm{SS}_{1}$ & $\mathrm{SS}_{2}^{1}$ & $\mathrm{SS}_{2}^{2}$ & $\mathrm{SS}_{3}^{1}$ & $\mathrm{SS}_{3}^{2}$ \\
\hline \multirow{4}{*}{$s_{1}^{i n}<M_{1}^{1}\left(D_{1}\right)$} & $s_{0}^{\text {in }}<F_{0}\left(D, s_{1}^{\text {in }}\right)$ & $\mathcal{J}_{1}$ & Cyan & $\mathrm{S}$ & & & & & \\
\hline & $F_{0}\left(D, s_{1}^{i n}\right)<s_{0}^{i n}<F_{2}^{1}(D)-s_{1}^{i n}$ & $\mathcal{J}_{2}$ & Green & $\mathrm{U}$ & S & & & & \\
\hline & $F_{2}^{1}(D)-s_{1}^{i n}<s_{0}^{i n}<F_{2}^{2}(D)-s_{1}^{i n}$ & $\mathcal{J}_{3}$ & Red & $\mathrm{U}$ & $\mathrm{U}$ & $\mathrm{S}$ & & & \\
\hline & $s_{0}^{\text {in }}>F_{2}^{2}(D)-s_{1}^{\text {in }}$ & $\mathcal{J}_{4}$ & Yellow & $\mathrm{U}$ & $\mathrm{S}$ & $\mathrm{S}$ & $\mathrm{U}$ & & \\
\hline \multirow{4}{*}{$M_{1}^{1}\left(D_{1}\right)<s_{1}^{i n}<M_{1}^{2}\left(D_{1}\right)$} & $s_{0}^{i n}>F_{2}^{2}(D)-s_{1}^{i n}$ & $\mathcal{J}_{5}$ & Yellow & $\mathrm{U}$ & $\mathrm{S}$ & $\mathrm{S}$ & $\mathrm{U}$ & $\mathrm{U}$ & \\
\hline & $F_{0}\left(D, s_{1}^{i n}\right)<s_{0}^{i n}<F_{2}^{2}(D)-s_{1}^{i n}$ & $\mathcal{J}_{6}$ & Red & $\mathrm{U}$ & $\mathrm{U}$ & S & & $\mathrm{U}$ & \\
\hline & $F_{1}^{1}(D)<s_{0}^{i n}<F_{0}\left(D, s_{1}^{i n}\right)$ & $\mathcal{J}_{7}$ & Red & $\mathrm{U}$ & & $\mathrm{S}$ & & $\mathrm{U}$ & \\
\hline & $s_{0}^{\text {in }}<F_{1}^{1}(D)$ & $\mathcal{J}_{8}$ & Blue & $\mathrm{U}$ & & & & $\mathrm{S}$ & \\
\hline \multirow{4}{*}{$M_{1}^{2}\left(D_{1}\right)<s_{1}^{i n}$} & $s_{0}^{i n}<F_{1}^{1}(D)$ & $\mathcal{J}_{9}$ & Deep pink & $\bar{S}$ & & & & $\mathrm{~S}$ & $\mathrm{U}$ \\
\hline & $F_{1}^{1}(D)<s_{0}^{i n}<F_{1}^{2}(D)$ & $\mathcal{J}_{10}$ & Gray & $\mathrm{S}$ & & $\mathrm{S}$ & & $\mathrm{U}$ & $\mathrm{U}$ \\
\hline & $F_{1}^{2}(D)<s_{0}^{i n}<F_{0}\left(D, s_{1}^{i n}\right)$ & $\mathcal{J}_{11}$ & Gray & $\mathrm{S}$ & & S & $\mathrm{U}$ & $\mathrm{U}$ & $\mathrm{U}$ \\
\hline & $s_{0}^{i n}>F_{0}\left(D, s_{1}^{i n}\right)$ & $\mathcal{J}_{12}$ & Yellow & $\mathrm{U}$ & S & $\mathrm{S}$ & $\mathrm{U}$ & $\mathrm{U}$ & $\mathrm{U}$ \\
\hline
\end{tabular}

Since the definition domain of the function $F_{i}^{j}$ is $I_{j}$ where $D \leq \bar{D}_{1}$ with $\Phi_{j}(D)>0$ (see Appen- 
dix A), it's necessary to distinguish the following two cases according to the sign of $\Phi_{j}\left(\bar{D}_{1}\right)$ :

$$
\text { case 1: } \Phi_{j}\left(\bar{D}_{1}\right)>0, \quad \text { case } 2: \Phi_{j}\left(\bar{D}_{1}\right) \leq 0 \text {. }
$$

Note that, the condition of case 1 is equivalent to $\left(\bar{\mu}_{0}\left(s_{1}^{\max }\right)-a_{0}\right) / \alpha_{0}>\bar{D}_{1}$ while the opposite inequality holds in case 2 .

Since it is very difficult to observe the twelve regions of the operating diagram in three-dimensional space, it would be much better to illustrate cuts along two-dimensional planes by fixing one of the three operating parameters in order to have a better vision and understanding. In subsection 4.1, we study the operating diagrams in the $\left(s_{1}^{i n}, s_{0}^{i n}\right)$ plane where $D$ is fixed. In subsection 4.2 , we determine the operating diagrams in the $\left(D, s_{0}^{i n}\right)$ plane where $s_{1}^{i n}$ is kept constant.

4.1. Operating diagrams in the $\left(s_{1}^{i n}, s_{0}^{i n}\right)$ plane when $D$ fixed. The intersection of the surface $\gamma_{0}$ with the $\left(s_{1}^{i n}, s_{0}^{i n}\right)$ plane where $D$ is kept constant is a curve of a function of $s_{1}^{i n}$. However, the intersections of the surfaces $\gamma_{i}^{j}, i=1,2,3, j=1,2$ with this plane are straight lines (see Table 10). The various regions of the operating diagram are then very clear to visualize it. To study the operating diagram when $D$ is fixed, we need the following result which determines the relative positions of the curve $\gamma_{0}$ with the straight lines $\gamma_{i}^{j}, i, j=1,2$ according to the values of $s_{1}^{i n}$ and $s_{1 j}^{i n *}$.
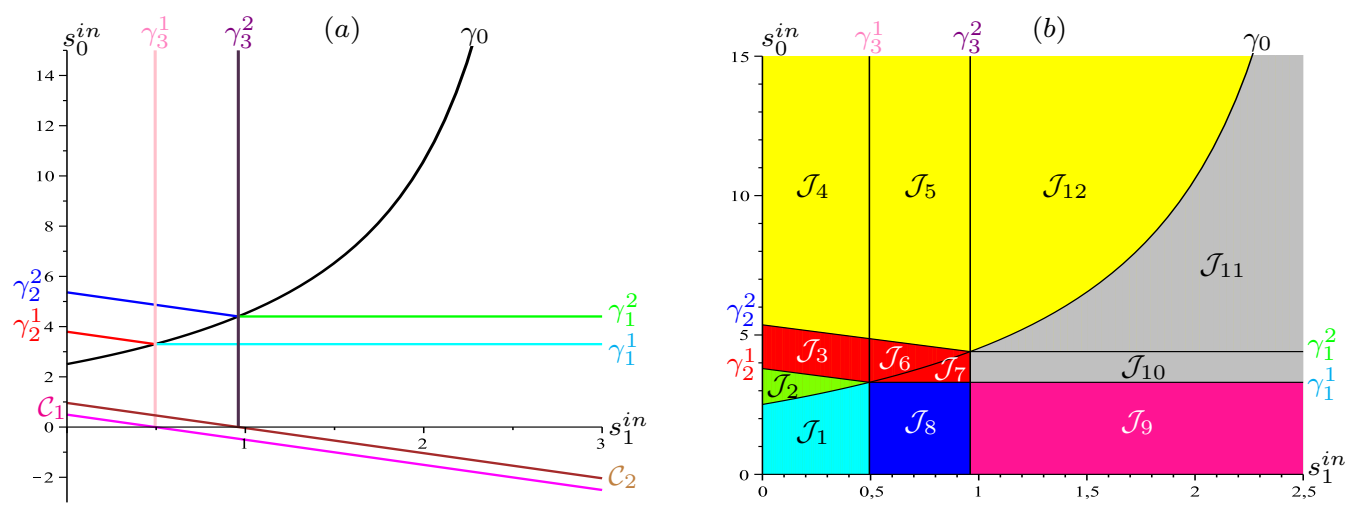

Figure 1. (a) The curves $\mathcal{C}_{1}, \mathcal{C}_{2}$ and those of $\Gamma$ in the case $D \in I_{2}=\left[0, \bar{D}_{1} \simeq 1.165\right)$ where $D=1.1<$ $\min \left(\bar{D}_{1}, \bar{D}_{0}(0) \simeq 2.556\right), s_{1}^{i n}=s_{11}^{i n *} \simeq 0.495$ and $s_{1}^{i n}=s_{12}^{i n *} \simeq 0.961$ and Case 1 of (4.1) holds. (b) The corresponding operating diagram in the $\left(s_{1}^{\text {in }}, s_{0}^{\text {in }}\right)$ plane.

(a)

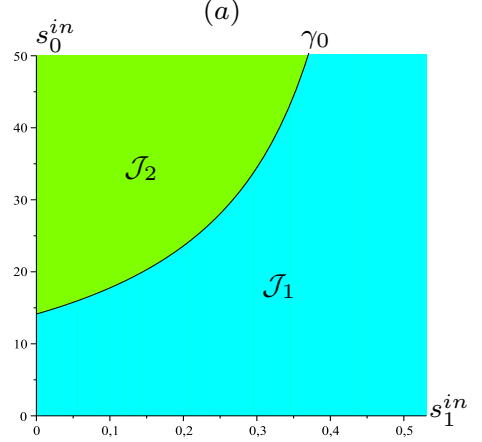

${ }^{(b)} \gamma_{3}^{2}$

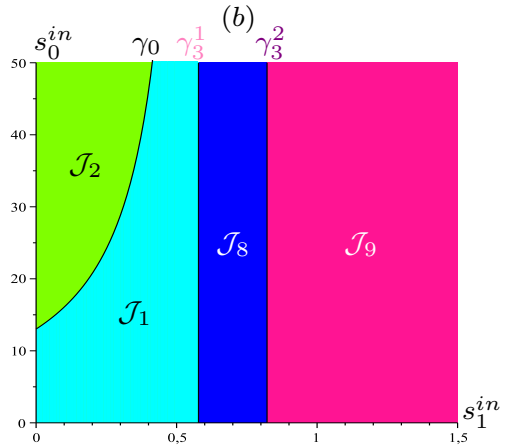

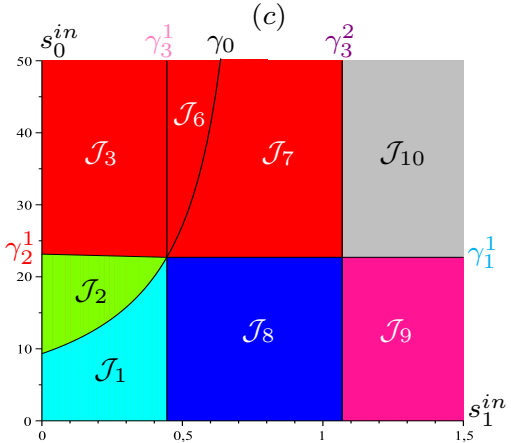

Figure 2. Operating diagrams in the $\left(s_{1}^{i n}, s_{0}^{i n}\right)$ plane with $D$ constant where case 2 of (4.1) holds: (a) $\bar{D}_{1} \simeq 1.856<$ $D=1.857<\bar{D}_{0}(0) \simeq 2.21$, (b) $\hat{D}_{1} \simeq 1.829<D=1.83<\bar{D}_{1}$, (c) $\widetilde{D}_{1} \simeq 1.285<D=1.7<\hat{D}_{1}$.

Proposition 4.2. Let $D \in \bar{I}_{j}$. We have $s_{1 j}^{i n *}:=M_{1}^{j}\left(D_{1}\right) \geq 0, j=1,2$ such that $s_{11}^{i n *}<s_{12}^{i n *}$. For all $D \in I_{1}$, the three curves $\gamma_{0}, \gamma_{1}^{1}$ and $\gamma_{2}^{1}$ intersect at the same point $s_{1}^{\text {in }}=s_{11}^{\text {in* }}$ (see Figures 1 and $2(c)$ ) such that $s_{11}^{i n *}<\bar{s}_{1}^{i n}$ where $\bar{s}_{1}^{i n}$ is the unique solution of $\bar{D}_{0}\left(s_{1}^{i n}\right)=D$. For all $s_{1}^{\text {in }} \in\left[0, s_{11}^{i n *}\right)$,

$$
F_{0}\left(D, s_{1}^{i n}\right)<F_{1}^{1}(D)<F_{2}^{1}(D)-s_{1}^{i n} .
$$

and for all $s_{1}^{\text {in }} \in\left(s_{11}^{i n *}, \bar{s}_{1}^{i n}\right)$,

$$
F_{0}\left(D, s_{1}^{i n}\right)>F_{1}^{1}(D)>F_{2}^{1}(D)-s_{1}^{i n}
$$


For all $D \in I_{2}$, the three curves $\gamma_{0}, \gamma_{1}^{2}$ and $\gamma_{2}^{2}$ intersect at the same point $s_{1}^{\text {in }}=s_{12}^{\text {in* }}$ (see Figure 1 ) such that $s_{12}^{\text {in* }}<\bar{s}_{1}^{\text {in }}$. For all $s_{1}^{\text {in }} \in\left[0, s_{12}^{\text {in* }}\right)$,

$$
F_{0}\left(D, s_{1}^{i n}\right)<F_{1}^{2}(D)<F_{2}^{2}(D)-s_{1}^{i n}
$$

and for all $s_{1}^{\text {in }} \in\left(s_{12}^{i n *}, \bar{s}_{1}^{i n}\right)$,

$$
F_{0}\left(D, s_{1}^{i n}\right)>F_{1}^{2}(D)>F_{2}^{2}(D)-s_{1}^{\text {in }} .
$$

According to the position of $D$ relatively to the critical values $\widetilde{D}_{i}, \hat{D}_{1}, \bar{D}_{1}$ and $\bar{D}_{0}(0)$ which are defined in Table 9 , the regions of the operating diagram in the $\left(s_{1}^{i n}, s_{0}^{i n}\right)$ plane where $D$ is kept constant are cataloged as follows:

1. if $D \in I_{2}$, then the twelve regions exist (see Figure 1);

2. if $D \in I_{1} \backslash I_{2}$, then eight regions exist where the four regions $\mathcal{J}_{4}, \mathcal{J}_{5}, \mathcal{J}_{11}$ and $\mathcal{J}_{12}$ are empty (see Figure 2(c));

3. if $D \in I_{0} \cap \bar{I}_{1} \backslash I_{1}$, or if $\hat{D}_{1}<D<\min \left(\bar{D}_{1}, \bar{D}_{0}(0)\right)$, then only the regions $\mathcal{J}_{1}, \mathcal{J}_{2}, \mathcal{J}_{8}$ and $\mathcal{J}_{9}$ exist (see Figure 2(b));

4. if $D \in \bar{I}_{1} \backslash\left\{I_{1} \cup I_{0}\right\}$, or if $\bar{D}_{0}(0)<D<\bar{D}_{1}$, then only the regions $\mathcal{J}_{1}, \mathcal{J}_{8}$ and $\mathcal{J}_{9}$ exist (see Figure 2(b) where $\mathcal{J}_{2}$ is empty);

5. if $D \in I_{0} \backslash \bar{I}_{1}$, or if $\bar{D}_{1}<D<\bar{D}_{0}(0)$, then only the regions $\mathcal{J}_{1}$ and $\mathcal{J}_{2}$ exist (see Figure 2(a));

6. if $D \notin I_{0} \cup \bar{I}_{1}$, or if $D>\max \left(\bar{D}_{1}, \bar{D}_{0}(0)\right)$, then only the region $\mathcal{J}_{1}$ exists (see Figure 2(a) where $\mathcal{J}_{2}$ is empty).

4.2. Operating diagrams in the $\left(D, s_{0}^{i n}\right)$ plane when $s_{1}^{\text {in }}$ fixed. The intersection of the surfaces $\gamma_{0}$ and $\gamma_{i}^{j}, i, j=1,2$ with the $\left(D, s_{0}^{i n}\right)$ plane where $s_{1}^{i n}$ is kept constant is a curve of a function of $D$. However, the intersections of the surfaces $\gamma_{3}^{j}, j=1,2$ with this plane are straight lines (see Table 11). To determine the operating diagram when $s_{1}^{\text {in }}$ is fixed, we show the following result which determines the relative positions of the curves $\gamma_{0}$ and $\gamma_{i}^{j}, i, j=1,2$ according to the values of $D$ and $D_{1}^{*}$. We begin by considering the case $s_{1}^{i n} \leq s_{1}^{\max }$.

Proposition 4.3. Let $s_{1}^{\text {in }} \leq s_{1}^{\max }$. For all $D \in I_{2}$,

$$
F_{0}\left(D, s_{1}^{i n}\right) \leq F_{1}^{2}(D) \leq F_{2}^{2}(D)-s_{1}^{i n} .
$$

There exists a solution $D=D_{1}^{*} \in \bar{I}_{1}$ of equation $s_{1}^{i n}=M_{1}^{1}\left(\alpha_{1} D+a_{1}\right)$ if and only if

$$
s_{1}^{\text {in }} \geq M_{1}^{1}\left(a_{1}\right) .
$$

It is unique if it exists. If $D_{1}^{*} \in I_{1}$, the three curves $\gamma_{0}, \gamma_{1}^{1}$ and $\gamma_{2}^{1}$ intersect at the same point $D=D_{1}^{*}$ (see Figures 3 and $4(b))$ such that for all $D \in\left[0, D_{1}^{*}\right)$, (4.3) holds and for all $D \in\left(D_{1}^{*}, \bar{D}_{1}\right) \cap I_{0} \cap I_{1}$, (4.2) holds. The curves $\gamma_{0}, \gamma_{1}^{1}$ and $\gamma_{2}^{1}$ do not intersect if $D_{1}^{*} \in \bar{I}_{1} \backslash I_{1}$, where for all $D \in I_{0},(4.3)$ holds (see Figure $4(c)$ ) or if $D_{1}^{*}$ does not exist, where for all $D \in I_{1},(4.2)$ holds (see Figures 3 and $4(a)$ ).

According to the position of $s_{1}^{\text {in }}$ relatively to $M_{1}^{1}\left(a_{1}\right)$ and the two cases of $(4.1)$ where $s_{1}^{\text {in }} \in\left[0, s_{1}^{\max }\right]$ and is kept constant, the regions of the operating diagram in the $\left(D, s_{0}^{\text {in }}\right)$ plane are cataloged as follows:

1. If $s_{1}^{\text {in }}<M_{1}^{1}\left(a_{1}\right)$, there exist at most four regions $\mathcal{J}_{1}$ to $\mathcal{J}_{4}$ (see Figure $3(\mathrm{a}, \mathrm{c})$ in case 1 of $(4.1)$ and Figure 4(a,d) in case 2).

2. Let $s_{1}^{\text {in }} \geq M_{1}^{1}\left(a_{1}\right)$. If $D_{1}^{*} \in I_{1}$, there exist at most eight regions $\mathcal{J}_{1}$ to $\mathcal{J}_{8}$ (see Figure $3(\mathrm{~b}, \mathrm{~d}$ ) in case 1 and Figure 4(b,e) in case 2). If case 2 holds and $D_{1}^{*} \in \bar{I}_{1} \backslash I_{1}$, there exist at most five regions $\mathcal{J}_{1}$ and $\mathcal{J}_{5}$ to $\mathcal{J}_{8}$ (see Figure $4(\mathrm{c}, \mathrm{f}$ )).

The operating diagram of Figure 4(b,e) shows the existence of seven regions $\mathcal{J}_{1}$ to $\mathcal{J}_{8}$ where the region $\mathcal{J}_{4}$ is empty in the case 2 of $(4.1)$ with $M_{1}^{1}\left(a_{1}\right)<s_{1}^{\text {in }} \leq s_{1}^{\max }$ and $D_{1}^{*} \in I_{1}$. However, this region $\mathcal{J}_{4}$ can be not empty for another set of parameters such that $D_{1}^{*}<\widetilde{D}_{1}$.

By similar arguments to that in the proof of Proposition 4.3, we can prove the following result which determines the relative positions of the curves $\gamma_{0}$ and $\gamma_{i}^{j}, i, j=1,2$ according to the values of $D$ and $D_{2}^{*}$ in the case $s_{1}^{i n}>s_{1}^{\max }$. 

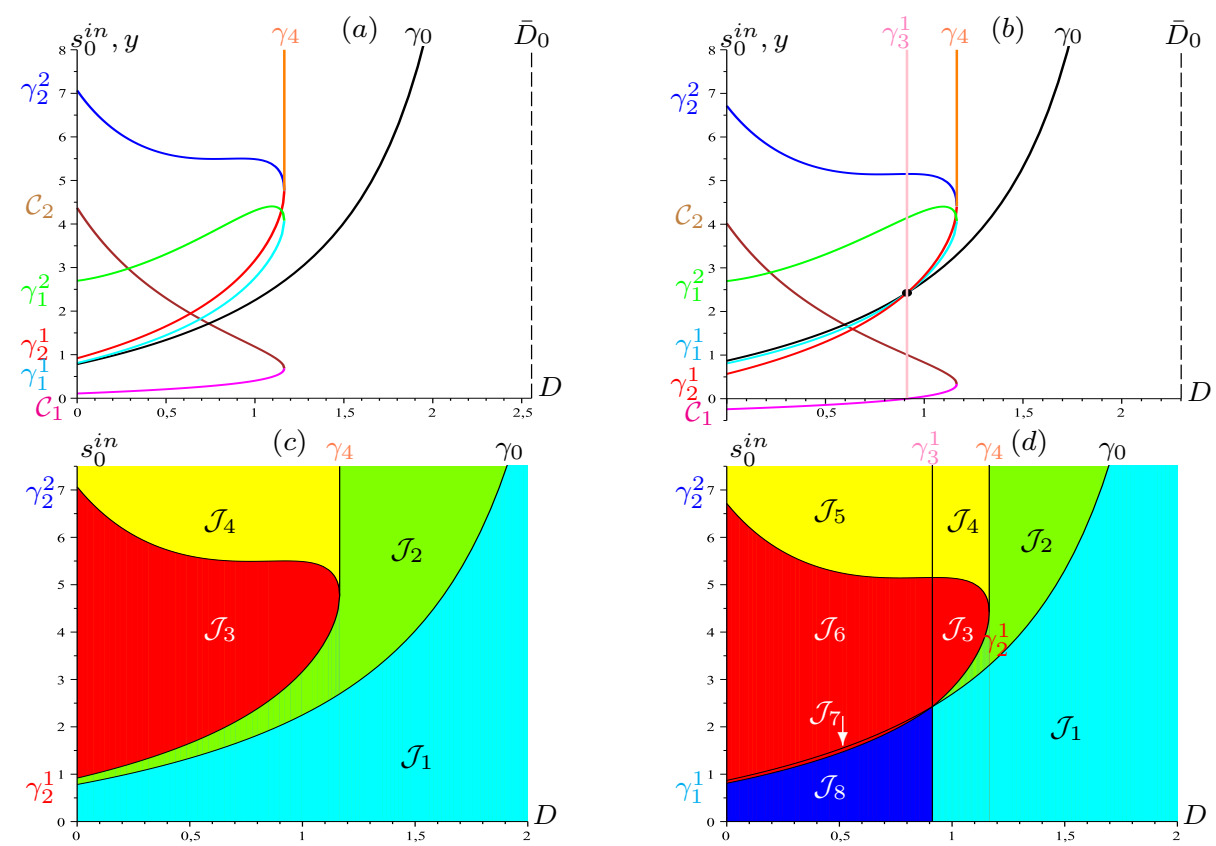

Figure 3. The curves $\mathcal{C}_{1}, \mathcal{C}_{2}$ and those of $\Gamma$ in case 1 of $(4.1)$ with $s_{1}^{\text {in }}<s_{1}^{\max } \simeq 0.689$ : (a) $s_{1}^{\text {in }}=0<M_{1}^{1}\left(a_{1}\right) \simeq 0.109$, (b) $M_{1}^{1}\left(a_{1}\right)<s_{1}^{i n}=0.35$. (c)-(d) The respective corresponding operating diagrams.
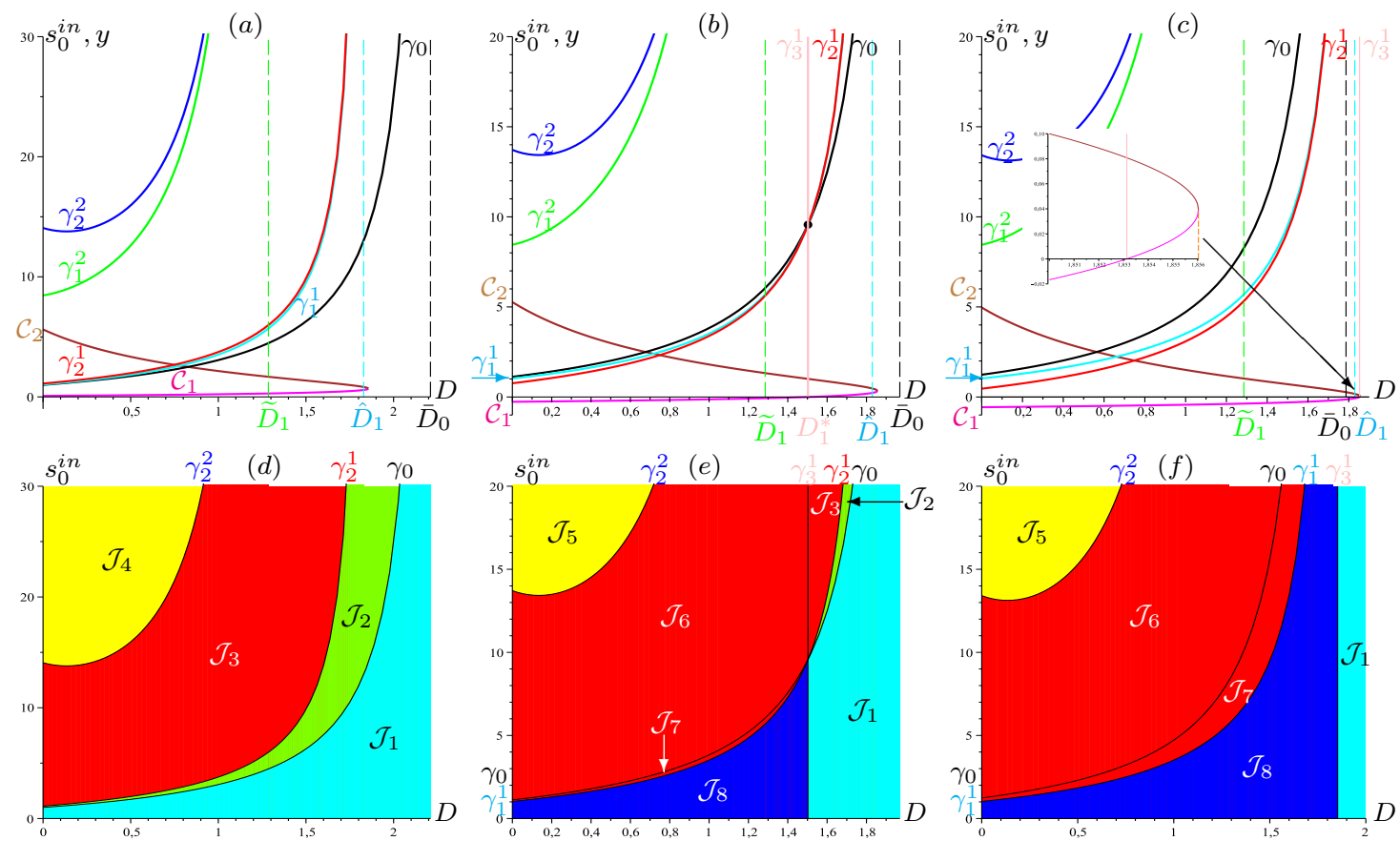

Figure 4. The curves $\mathcal{C}_{j}, j=1,2$ and those of $\Gamma$ in case 2 of $(4.1)$ with $s_{1}^{\text {in }}<s_{1}^{\text {max }} \simeq 0.689$ : (a) $s_{1}^{\text {in }}=0<$ $M_{1}^{1}\left(a_{1}\right) \simeq 0.109$, (b) $M_{1}^{1}\left(a_{1}\right)<s_{1}^{i n}=0.35 ; D_{1}^{*} \simeq 1.503 \in I_{1}, \hat{D}_{1} \simeq 1.829$ (c) $M_{1}^{1}\left(a_{1}\right)<s_{1}^{i n}=0.65 ; D_{1}^{*} \simeq 1.853 \notin I_{1}$, and a magnification of $\mathcal{C}_{j}$ when $D \in\left[1.85, \bar{D}_{1}\right], \bar{D}_{1} \simeq 1.856$. (d)-(e)-(f) The respective corresponding operating diagrams.

Proposition 4.4. Let $s_{1}^{\text {in }}>s_{1}^{\max }$. For all $D \in I_{0} \cap I_{1}$, we have

$$
F_{2}^{1}(D)-s_{1}^{i n}<F_{1}^{1}(D)<F_{0}\left(D, s_{1}^{i n}\right) .
$$

There exists a solution $D=D_{2}^{*} \in \bar{I}_{2}$ of equation $s_{1}^{i n}=M_{1}^{2}\left(\alpha_{1} D+a_{1}\right)$ if and only if

$$
s_{1}^{i n} \leq M_{1}^{2}\left(a_{1}\right) .
$$

It is unique if it exists. If $D_{2}^{*} \in I_{2}$, the three curves $\gamma_{0}, \gamma_{1}^{2}$ and $\gamma_{2}^{2}$ intersect at the same point $D=D_{2}^{*}$ (see Figure 5(a) and Figure 6(b)) such that for all $D \in\left[0, D_{2}^{*}\right) \cap I_{2}$, (4.4) holds and for all $D \in\left(D_{2}^{*}, \bar{D}_{1}\right) \cap I_{0} \cap I_{2}$, (4.5) holds. The curves $\gamma_{0}, \gamma_{1}^{2}$ and $\gamma_{2}^{2}$ do not intersect if $D_{2}^{*} \in \bar{I}_{2} \backslash I_{2}$, where for all $D \in I_{2}$, (4.4) holds (see Figure 6(a)) or if $D_{2}^{*}$ does not exist, where for all $D \in I_{2}$, (4.5) holds (see Figure 5(b) and Figure 6(c)). 

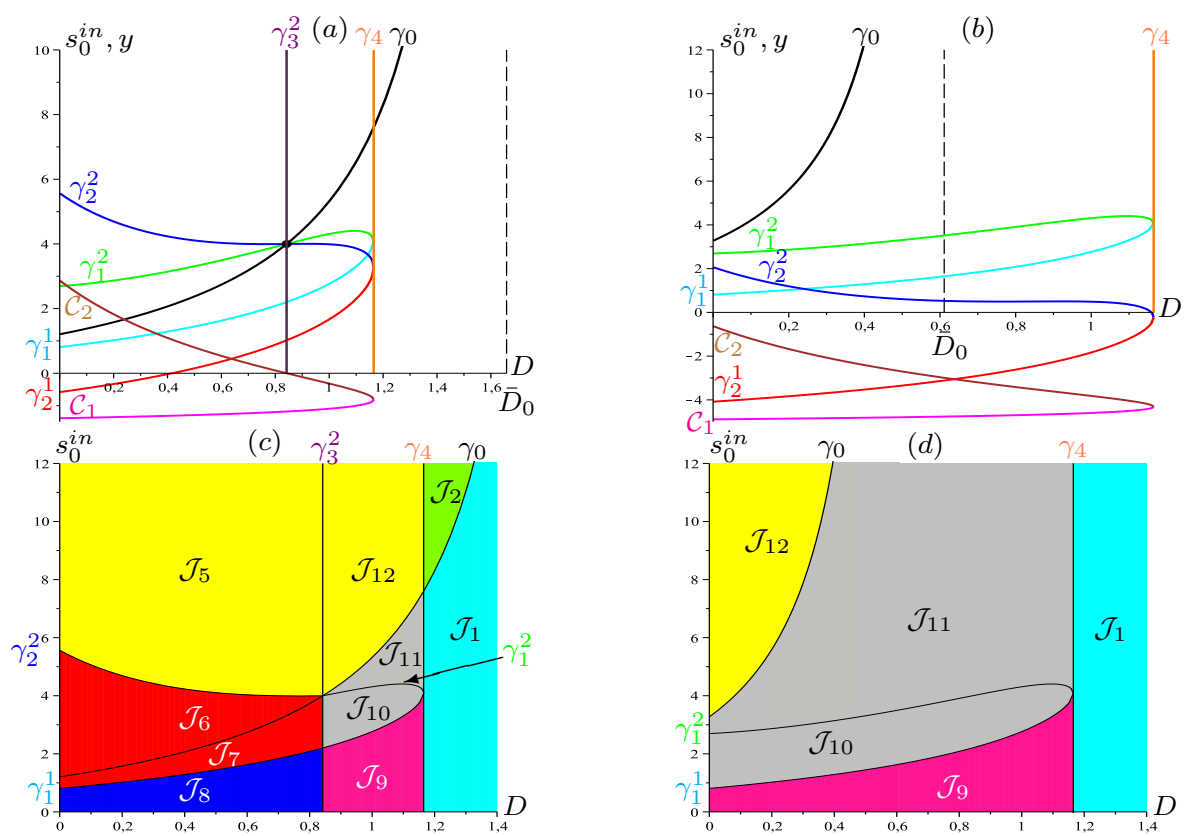

Figure 5. The curves $\mathcal{C}_{1}, \mathcal{C}_{2}$ and those of $\Gamma$ in case 1 of $(4.1)$ with $s_{1}^{\text {in }}>s_{1}^{\text {max }} \simeq 0.689$ : (a) $s_{1}^{\text {in }}=1.5<M_{1}^{2}\left(a_{1}\right) \simeq$ 4.37, (b) $s_{1}^{i n}=5>\max \left(s_{1}^{\max }, M_{1}^{2}\left(a_{1}\right)\right)$. (c)-(d) The respective corresponding operating diagrams.
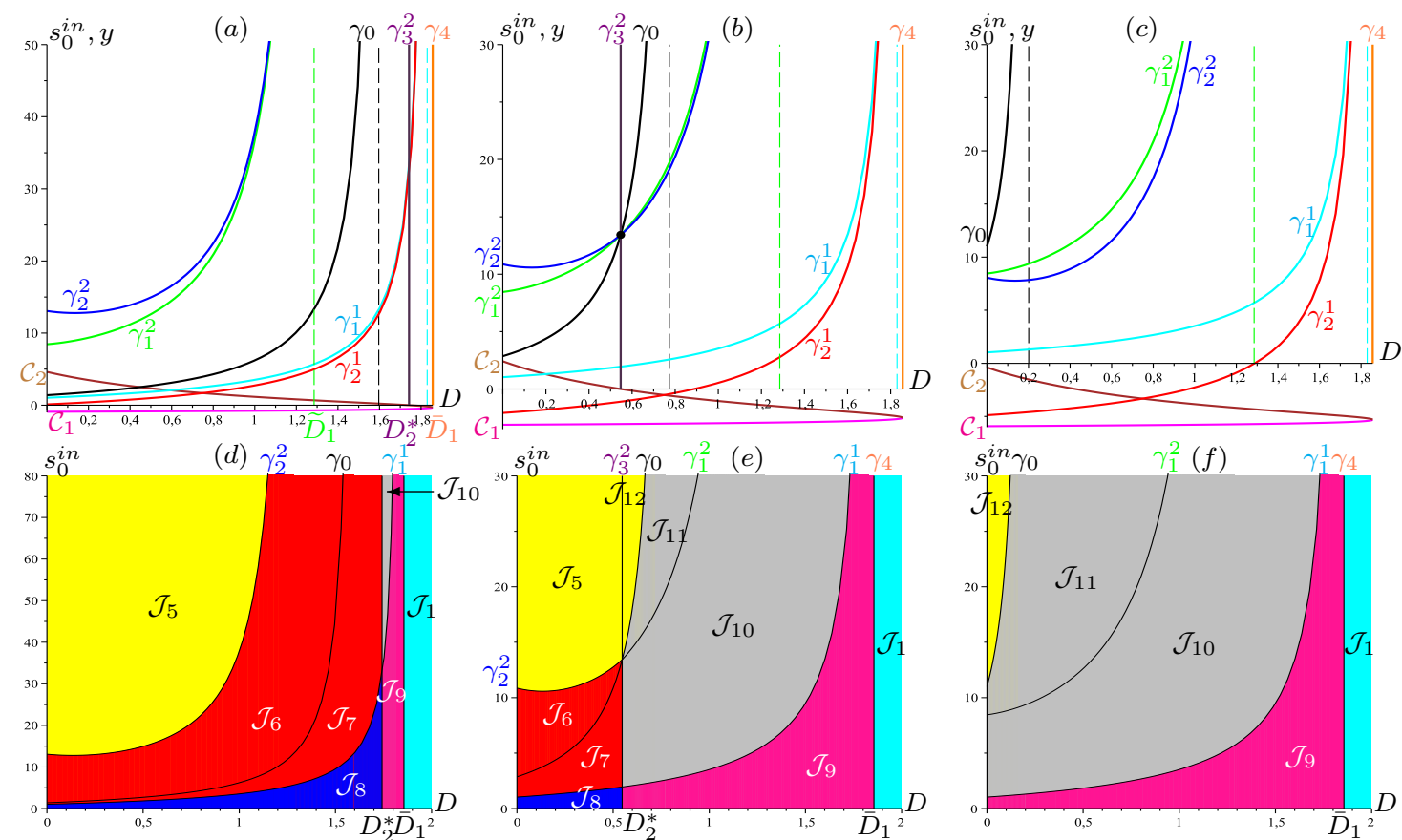

Figure 6. The curves $\mathcal{C}_{1}, \mathcal{C}_{2}$ and those of $\Gamma$ in case 2 of (4.1) with $s_{1}^{\text {in }}>s_{1}^{\text {max }} \simeq 0.689$ : (a) $s_{1}^{\text {in }}=1<M_{1}^{2}\left(a_{1}\right) \simeq 5.615$; $D_{2}^{*} \simeq 1.742 \notin I_{2}=[0,1.285)$, (b) $s_{1}^{i n}=3.2<M_{1}^{2}\left(a_{1}\right) ; D_{2}^{*} \in I_{2}$, (c) $s_{1}^{i n}=6>M_{1}^{2}\left(a_{1}\right)$. (d)-(e)-(f) The respective corresponding operating diagrams.

According to the position of $s_{1}^{\text {in }}$ relatively to $M_{1}^{2}\left(a_{1}\right)$ and the two cases of (4.1) where $s_{1}^{\text {in }}>s_{1}^{\max }$ and is kept constant, the regions of the operating diagram in the $\left(D, s_{0}^{i n}\right)$ plane are cataloged as follows:

1. Let $s_{1}^{\text {in }} \leq M_{1}^{2}\left(a_{1}\right)$. If $D_{2}^{*} \in I_{2}$, in case 1 of (4.1), $\mathcal{J}_{3}$ and $\mathcal{J}_{4}$ are empty and the other ten regions can exist (see Figure $5(\mathrm{a}, \mathrm{c})$ ), while in case $2, \mathcal{J}_{2}$ to $\mathcal{J}_{4}$ are empty and we can have up to nine regions in the operating diagram (see Figure 6(b,e) in case 2). If case 2 holds and $D_{2}^{*} \in \bar{I}_{2} \backslash I_{2}$, there exist at most seven regions $\mathcal{J}_{1}$ and $\mathcal{J}_{5}$ to $\mathcal{J}_{10}$ (see Figure $6(\mathrm{a}, \mathrm{d})$ ).

2. If $s_{1}^{\text {in }}>M_{1}^{2}\left(a_{1}\right)$, at most the five regions $\mathcal{J}_{1}$ and $\mathcal{J}_{9}$ to $\mathcal{J}_{12}$ can exist (see Figure $5(\mathrm{~b}, \mathrm{~d})$ in case 1 and Figure 6(c,f) in case 2).

With the same set of parameters of the operating diagram of Figure 3(a) that we redraw in Figure 7(a), the equation $\Phi_{2}(D)=0$ has no solution. In Appendix C, with the specific growth rates defined in (C.1), 
we show that this equation has at most three solutions $\widetilde{D}_{j}, j=1,2,3$ such that $\lim _{D \rightarrow \widetilde{D}_{j}^{-}} F_{i}^{2}(D)=+\infty$, $i=1,2$ (see Proposition A.4). The operating diagrams of Figure 7(b-c) show that in the case of two or three solutions of the equation $\Phi_{2}(D)=0$, we have the same number of regions but there is a change in the shape and the connectivity of the regions. This property is the same for the various cases studied above even when the equation $\Phi_{2}(D)=0$ has several roots with general growth rates satisfying Hypotheses 2.1 to 2.4 .
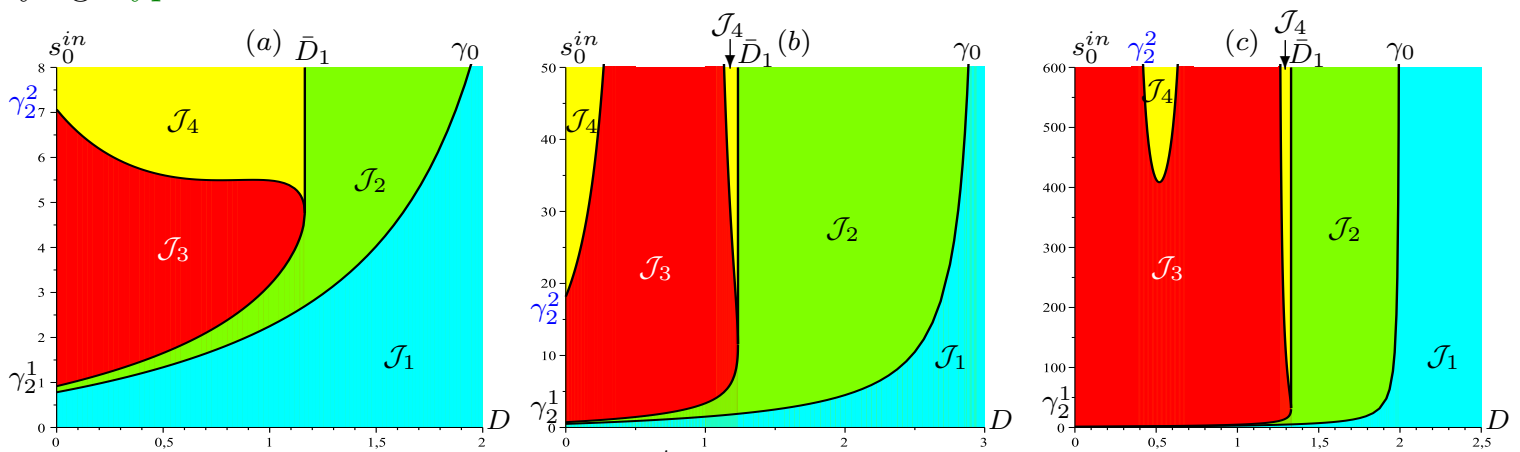

Figure 7. Operating diagrams of ${ }^{2}(2.1)$ when $s_{1}^{\text {in }} \stackrel{1}{=} 0$ and case 1 of ${ }^{3}(4.1)$ holds: equation $\Phi_{2}^{1,5}(D) \stackrel{2}{=} 0\left({ }^{2,5}\right)$ has no solution, (b) has two solutions (c) has three solutions. The operating diagram (a) is the same in Figure 3(c).

The following result determines the nature of bifurcations of system (2.1) that might happen by crossing the various regions of the operating parameters space $\left(D, s_{0}^{i n}, s_{1}^{i n}\right)$ through the surfaces of $\Gamma$ where the steady states coalesce and can change stability.

Proposition 4.5. The bifurcation analysis of the steady states of (2.1) by crossing the surfaces of $\Gamma$ according to the operating parameters $D, s_{0}^{\text {in }}$ and $s_{1}^{\text {in }}$ is summarized in Table 8.

Table 8

Bifurcations according to surfaces of $\Gamma$. The letter TB (resp. SNB) means a transcritical bifurcation (resp. saddlenode bifurcation). Note that $k=2,3$.

\begin{tabular}{|c|c|c|c|}
\hline$\Gamma$ & Conditions & Transition & Bifurcation \\
\hline \multirow{3}{*}{$\gamma_{0}$} & $s_{1}^{i n}<M_{1}^{1}\left(D_{1}\right)$ & $\mathcal{J}_{1}$ to $\mathcal{J}_{2}$ & \multirow{3}{*}{$\mathrm{TB}: \mathrm{SS}_{0}=\mathrm{SS}_{1}$} \\
\hline & $M_{1}^{1}\left(D_{1}\right)<s_{1}^{i n}<M_{1}^{2}\left(D_{1}\right)$ & $\mathcal{J}_{6}$ to $\mathcal{J}_{7}$ & \\
\hline & $M_{1}^{2}\left(D_{1}\right)>s_{1}^{i n}$ & $\mathcal{J}_{11}$ to $\mathcal{J}_{12}$ & \\
\hline \multirow{2}{*}{$\gamma_{1}^{1}$} & $M_{1}^{1}\left(D_{1}\right)<s_{1}^{i n}<M_{1}^{2}\left(D_{1}\right)$ & $\mathcal{J}_{7}$ to $\mathcal{J}_{8}$ & \multirow{2}{*}{$\mathrm{TB}: \mathrm{SS}_{2}^{1}=\mathrm{SS}_{3}^{1}$} \\
\hline & $s_{1}^{i n}>M_{1}^{2}\left(D_{1}\right)$ & $\mathcal{J}_{9}$ to $\mathcal{J}_{10}$ & \\
\hline$\gamma_{1}^{2}$ & $s_{1}^{\text {in }}>M_{1}^{2}\left(D_{1}\right)$ & $\mathcal{J}_{10}$ to $\mathcal{J}_{11}$ & TB: $\mathrm{SS}_{2}^{2}=\mathrm{SS}_{3}^{2}$ \\
\hline$\gamma_{2}^{1}$ & $s_{1}^{\text {in }}<M_{1}^{1}\left(D_{1}\right)$ & $\mathcal{J}_{2}$ to $\mathcal{J}_{3}$ & TB: $\mathrm{SS}_{1}=\mathrm{SS}_{2}^{1}$ \\
\hline \multirow{2}{*}{$\gamma_{2}^{2}$} & $s_{1}^{i n}<M_{1}^{1}\left(D_{1}\right)$ & $\mathcal{J}_{3}$ to $\mathcal{J}_{4}$ & \multirow{2}{*}{$\mathrm{TB}: \mathrm{SS}_{1}=\mathrm{SS}_{2}^{2}$} \\
\hline & $M_{1}^{1}\left(D_{1}\right)<s_{1}^{i n}<M_{1}^{2}\left(D_{1}\right)$ & $\mathcal{J}_{5}$ to $\mathcal{J}_{6}$ & \\
\hline \multirow{3}{*}{$\gamma_{3}^{1}$} & $s_{0}^{i n}>F_{2}^{2}-s_{1}^{i n}$ & $\mathcal{J}_{4}$ to $\mathcal{J}_{5}$ & \multirow{3}{*}{$\mathrm{TB}: \mathrm{SS}_{0}=\mathrm{SS}_{3}^{1}$} \\
\hline & $F_{2}^{1}-s_{1}^{i n}<s_{0}^{i n}<F_{2}^{2}-s_{1}^{i n}$ if $D<D_{1}^{*}$, if not $F_{0}<s_{0}^{i n}<F_{2}^{2}-s_{1}^{i n}$ & $\mathcal{J}_{3}$ to $\mathcal{J}_{6}$ & \\
\hline & $s_{0}^{i n}<F_{0}$ if $D<D_{1}^{*}$, if not $s_{0}^{i n}<F_{1}^{1}$ & $\mathcal{J}_{1}$ to $\mathcal{J}_{8}$ & \\
\hline \multirow{3}{*}{$\gamma_{3}^{2}$} & $s_{0}^{i n}>F_{2}^{2}-s_{1}^{i n}$ if $D<D_{2}^{*}$, if not $s_{0}^{i n}>F_{0}$ & $\mathcal{J}_{5}$ to $\mathcal{J}_{12}$ & \multirow{3}{*}{$\mathrm{TB}: \mathrm{SS}_{0}=\mathrm{SS}_{3}^{2}$} \\
\hline & $F_{1}^{1}<s_{0}^{i n}<F_{0}$ if $D<D_{2}^{*}$, if not $F_{1}^{1}<s_{0}^{i n}<F_{1}^{2}$ & $\mathcal{J}_{7}$ to $\mathcal{J}_{10}$ & \\
\hline & $s_{0}^{\text {in }}<F_{1}^{1}$ & $\mathcal{J}_{8}$ to $\mathcal{J}_{9}$ & \\
\hline$\gamma_{4}$ & $s_{1}^{i n}<s_{1}^{\max }$ & $\mathcal{J}_{2}$ to $\mathcal{J}_{4}$ & SNB: $\mathrm{SS}_{2}^{1}=\mathrm{SS}_{2}^{2}$ \\
\hline & \multirow{3}{*}{$s_{1}^{i n}>s_{1}^{\max }$} & $\mathcal{J}_{2}$ to $\mathcal{J}_{12}$ & SNB: $\mathrm{SS}_{k}^{1}=\mathrm{SS}_{k}^{2}$ \\
\hline & & $\mathcal{J}_{1}$ to $\mathcal{J}_{11}$ & \\
\hline & & $\mathcal{J}_{1}$ to $\mathcal{J}_{9}$ & SNB: $\mathrm{SS}_{3}^{1}=\mathrm{SS}_{3}^{2}$ \\
\hline
\end{tabular}

We have only studied the bifurcations that occur by transitions through surfaces in two-dimensional planes and not through the points given by the intersections of curves and lines. However, the study of such bifurcations can be determined in the same way.

5. Discussion and conclusion. In this paper, we have generalized the mathematical analysis of the simplified model (2.1) of anaerobic digestion in the form of a two-tiered microbial food chain describing a syntrophic relationship between two microbial species in a chemostat. To this end, we allow a large class of growth functions with distinct disappearance rates. The main contribution of this 
study is to bring out the mutual effects of the syntrophy relationship, the decay of the two microbial species, the substrate inhibition on the growth of the second species and a new inflowing concentration (hydrogen) which are not studied together in the existing literature. First, we have determined the necessary and sufficient conditions of existence and local stability of all steady states of syntrophic model (2.1) according to the operating parameters $D, s_{0}^{i n}$ and $s_{1}^{i n}$. Second, we have analyzed the operating diagrams to determine the behavior of the system according to the control parameters and to choose the appropriate inputs and the initial states to achieve a good operation of the process. The operating diagrams show that the system can have a unique stable steady state: either of coexistence $\left(\mathcal{J}_{i}, i=3,6,7\right)$ or washout $\left(\mathcal{J}_{1}\right)$ or exclusion of one of two microbial species $\left(\mathcal{J}_{i}, i=2,8\right)$. It can also exhibit a bistability between coexistence and washout $\left(\mathcal{J}_{i}, i=10,11\right)$ or exclusion of the second species $\left(\mathcal{J}_{i}, i=4,5,12\right)$ or between washout and exclusion of the first species $\left(\mathcal{J}_{9}\right)$. If required, to ensure (or to avoid) the coexistence of two microbial species in the process, the operating parameter values can be chosen in (out) the regions $\mathcal{J}_{i}, i=3,6,7$, where there exists a unique stable steady state of coexistence and the other steady states are unstable. The study of the nature of bifurcations of the steady states shows that all the coalesces and the change of stability can be either by a transcritical or a saddle-node bifurcation by crossing the boundary of the regions of the operating parameters space.

In [40], where $s_{1}^{i n}=0$ and $\mu_{1}$ is increasing, the analysis of the operating diagram of (2.1) shows the existence only of the three regions $\mathcal{J}_{1}$ to $\mathcal{J}_{3}$ where the bistability cannot occur and the two steady states of extinction of the first species $\mathrm{SS}_{3}^{1}$ and $\mathrm{SS}_{3}^{2}$ do not exist. In [18], where only $s_{1}^{i n}=0$, that is, $\mu_{1}$ is nonmonotonic, $\mathrm{SS}_{3}^{1}$ and $\mathrm{SS}_{3}^{2}$ do not exist and the operating diagram has at most four regions $\mathcal{J}_{1}$ to $\mathcal{J}_{4}$ where the system can exhibit a bistability between the steady state $\mathrm{SS}_{1}$ of exclusion of the second species and the coexistence steady state $\mathrm{SS}_{2}^{1}$. It is shown, when the substrate inhibition increases, that there is an emergence of the bistability region $\mathcal{J}_{4}$ first and then its disappearance with the coexistence region $\mathcal{J}_{3}$ for a sufficiently large substrate inhibition rate.

Conversely in [11], where $s_{1}^{\text {in }} \geq 0$ and $\mu_{1}$ is increasing, it is shown that the steady states of coexistence $\mathrm{SS}_{2}$ and of extinction of the first species $\mathrm{SS}_{3}$ are unique. Moreover, the bistability cannot occur where at most six regions exist such that all bistability regions $\mathcal{J}_{4}, \mathcal{J}_{5}$ and $\mathcal{J}_{9}$ to $\mathcal{J}_{12}$ do not exist. Thus, our mathematical study of the operating diagrams of model (2.1) shows the significant impact of substrate inhibition on the behavior of the process and the emergence of the bistability regions which are empty when the growth rate $\mu_{1}$ is increasing $[11,40]$. Our findings on the destabilization of a twotiered microbial 'food chain' by substrate inhibition are similar to those in $[18,24]$ where the behavior of system depends on the initial condition. Furthermore, a low, as well as a high concentration of input substrate, can cause destabilization by the extinction of the highest trophic level of a tri-trophic food chain model in the chemostat [8].

Recently in [38], an extension of the study of the two-tiered model (1.2) in [5] with a commensalistic relationship $\left(\mu_{0}(\cdot)=\mu_{0}\left(s_{0}\right)\right)$ provides a complete analysis of the operating diagram. It is shown that our six steady states exist and at most nine regions exist where only the regions $\mathcal{J}_{7}, \mathcal{J}_{10}$ and $\mathcal{J}_{11}$ are empty. Hence, the main change of the behavior of the process by considering the effect of syntrophic relationship compared to [38] is that the system can exhibit a bistability between the washout $\mathrm{SS}_{0}$ and the coexistence $\mathrm{SS}_{2}^{1}$.

These theoretical messages explain the joined effect of syntrophy, mortality, substrate inhibition, and input substrates on the maintenance of coexistence and the protection of microbial ecosystems. Finally, the results in this contribution may also serve for optimal experimental design by studying the biogas production and the process performance with respect to operating parameters. This is an important question that deserves further attention and will be the object of future work.

Appendix A. Definition domains and properties of auxiliary functions. First, we introduce in Table 9 some notations and the definition domains of the auxiliary functions defined in Table 3 that we will show in this section. The following proposition determines the domain and some properties of the function $F_{0}\left(D, s_{1}^{i n}\right)$.

Proposition A.1. The function $F_{0}\left(D, s_{1}^{i n}\right)$ is defined for $\left(D, s_{1}^{i n}\right) \in \operatorname{dom}\left(F_{0}\right)$. Moreover, one has 
Table 9

Notations and intervals of auxiliary functions.

Definition

\begin{tabular}{|c|c|}
\hline $\begin{array}{l}\bar{D}_{0}\left(s_{1}^{i n}\right) \\
\bar{D}_{1}\end{array}$ & $\begin{array}{l}\bar{D}_{0}\left(s_{1}^{i n}\right)=\left(\bar{\mu}_{0}\left(s_{1}^{i n}\right)-a_{0}\right) / \alpha_{0} \\
\bar{D}_{1}=\left(\mu_{1}^{\max }-a_{1}\right) / \alpha_{1}\end{array}$ \\
\hline$\Phi_{j}(D)$ & $\Phi_{j}(D)=\bar{\mu}_{0}\left(M_{1}^{j}\left(D_{1}\right)\right)-D_{0}, \quad$ for $D \in \bar{I}_{j}, j=1,2$ \\
\hline$\hat{D}_{1}$ & $\hat{D}_{1}$ is the solution of $\Phi_{1}(D)=0$ \\
\hline$\widetilde{D}_{i}$ & $\widetilde{D}_{i}$ are the solutions of $\Phi_{2}(D)=0, i=1, \ldots, n$ \\
\hline$I_{0}$ & $\begin{array}{l}\text { Let } s_{1}^{\text {in }} \geqslant 0 . I_{0}=\left[0, \bar{D}_{0}\left(s_{1}^{i n}\right)\right) \text { if } \alpha_{0} \in(0,1] \\
I_{0}=[0,+\infty) \text { if } \alpha_{0}=0 \text { and } a_{0}<\bar{\mu}_{0}\left(s_{1}^{i n}\right), \text { otherwise } I_{0}=\emptyset\end{array}$ \\
\hline$J_{0}$ & $\begin{array}{l}\text { Let } D \geqslant 0 . J_{0}=\left[0, \bar{s}_{1}^{i n}\right), \text { if } \bar{D}_{0}(+\infty)<D<\bar{D}_{0}(0) \\
\text { where } \bar{s}_{1}^{i n} \text { is the unique solution of } \bar{D}_{0}\left(s_{1}^{i n}\right)=D \\
J_{0}=[0,+\infty), \text { if } D \leq \bar{D}_{0}(+\infty) \text { and } J_{0}=\emptyset \text { if } D \geq \bar{D}_{0}(0)\end{array}$ \\
\hline $\operatorname{dom}\left(F_{0}\right)$ & $\operatorname{dom}\left(F_{0}\right)=\left\{\left(D, s_{1}^{i n}\right):\left(D \in I_{0}\right.\right.$ and $\left.s_{1}^{i n} \geq 0\right)$ or $\left(s_{1}^{i n} \in J_{0}\right.$ and $\left.\left.D \geq 0\right)\right\}$ \\
\hline $\bar{I}_{1}$ & $\begin{array}{l}\bar{I}_{1}=\left[0, \bar{D}_{1}\right] \text { if } \alpha_{1} \in(0,1] \\
\bar{I}_{1}=[0,+\infty) \text { if } \alpha_{1}=0 \text { and } a_{1} \leq \mu_{1}^{\max }, \text { otherwise } \bar{I}_{1}=\emptyset\end{array}$ \\
\hline$\overline{\bar{I}_{2}}$ & $\bar{I}_{2}=\bar{I}_{1}$ if $a_{1}>0$ and $\bar{I}_{2}=\bar{I}_{1} \backslash\{0\}$ if $a_{1}=0$ \\
\hline$I_{j}$, & $I_{j}=\left\{D \in \bar{I}_{j} / \Phi_{j}(D)>0\right\}, j=1,2$ \\
\hline
\end{tabular}

$\bar{D}_{1}<\bar{D}_{0}(0)$ in case 1 of (4.1), $\hat{D}_{1}<\bar{D}_{0}(0)$ in case 2 of (4.1) and

$$
\lim _{s_{1}^{i n} \rightarrow \bar{s}_{1}^{i n-}} F_{0}\left(D, s_{1}^{i n}\right)=+\infty .
$$

Proof. Let $s_{1}^{i n}$ be fixed. From Table 3, the function $F_{0}\left(D, s_{1}^{i n}\right)$ is defined if and only if

$$
D_{0}<\bar{\mu}_{0}\left(s_{1}^{i n}\right) \quad \Longleftrightarrow \quad D<\bar{D}_{0}\left(s_{1}^{i n}\right)=\frac{\bar{\mu}_{0}\left(s_{1}^{i n}\right)-a_{0}}{\alpha_{0}},
$$

where $\bar{D}_{0}\left(s_{1}^{i n}\right)$ is defined and positive if and only if $\alpha_{0} \neq 0$ and $\bar{\mu}_{0}\left(s_{1}^{i n}\right)>a_{0}$. In the particular case $\alpha_{0}=0$, the condition $D_{0}<\bar{\mu}_{0}\left(s_{1}^{i n}\right)$ is equivalent to $a_{0}<\bar{\mu}_{0}\left(s_{1}^{i n}\right)$. Thus, the function $F_{0}\left(\cdot, s_{1}^{i n}\right)$ is defined on $I_{0}$. Let $D$ be fixed. From Hypothesis 2.5, the function $s_{1}^{i n} \mapsto \bar{D}_{0}\left(s_{1}^{i n}\right)$ is decreasing from $\bar{D}_{0}(0)$ to $\bar{D}_{0}(+\infty)$. If $D \leq \bar{D}_{0}(+\infty)$, then (A.1) holds for all $s_{1}^{\text {in }} \geq 0$. If $\bar{D}_{0}(+\infty)<D<\bar{D}_{0}(0)$, then there exists a unique solution $\bar{s}_{1}^{i n}$ of equation $D=\bar{D}_{0}\left(s_{1}^{i n}\right)$ such that (A.1) holds for all $s_{1}^{i n} \in\left[0, \bar{s}_{1}^{i n}\right)$. If $D \geq \bar{D}_{0}(0)$, (A.1) does not hold for all $s_{1}^{i n} \geq 0$. Hence, the function $F_{0}(D, \cdot)$ is defined on $J_{0}$. Consequently, $F_{0}\left(D, s_{1}^{i n}\right)$ is defined for $\left(D, s_{1}^{i n}\right) \in \operatorname{dom}\left(F_{0}\right)$. Since $\bar{s}_{1}^{i n}$ satisfies $D_{0}=\mu_{0}\left(+\infty, \bar{s}_{1}^{i n}\right)$, it follows that

$$
F_{0}\left(D, \bar{s}_{1}^{i n}\right)=M_{0}\left(\mu_{0}\left(+\infty, \bar{s}_{1}^{i n}\right), \bar{s}_{1}^{i n}\right)=+\infty .
$$

When case 1 of (4.1) holds, we have

$$
\alpha_{0} \bar{D}_{1}+a_{0}<\bar{\mu}_{0}\left(M_{1}^{1}\left(\alpha_{1} \bar{D}_{1}+a_{1}\right)\right)<\bar{\mu}_{0}(0),
$$

because the function $\bar{\mu}_{0}(\cdot)$ is decreasing (see Hypothesis 2.5). Thus, $\bar{D}_{1}<\bar{D}_{0}(0)$. Moreover, when case 2 of (4.1) holds, we conclude that $\hat{D}_{1}<\bar{D}_{0}(0)$ where $\hat{D}_{1}$ is a solution of $\Phi_{1}(D)=0$ because

$$
\alpha_{0} \hat{D}_{1}+a_{0}=\bar{\mu}_{0}\left(M_{1}^{1}\left(\alpha_{1} \hat{D}_{1}+a_{1}\right)\right)<\bar{\mu}_{0}(0) .
$$

The following result determines the definition domain $I_{1}$ of the function $F_{i}^{1}(\cdot), i=1,2$, according to the coefficients $\alpha_{0}$ and $\alpha_{1}$.

Proposition A.2. Let $s_{1}^{i n} \geq 0$ be fixed. The function $F_{i}^{1}(D), i=1,2$, is defined on

$$
I_{1}= \begin{cases}{[0,+\infty),} & \text { if } \alpha_{0}=\alpha_{1}=0, a_{1}<\mu_{1}^{\max }, \bar{\mu}_{0}\left(M_{1}^{1}\left(a_{1}\right)\right)>a_{0} \\ \emptyset, & \text { if } \alpha_{0}=\alpha_{1}=0 \text { and } a_{1} \geq \mu_{1}^{\max } \text { or } \bar{\mu}_{0}\left(M_{1}^{1}\left(a_{1}\right)\right) \leq a_{0} \\ {\left[0,\left(\bar{\mu}_{0}\left(M_{1}^{1}\left(a_{1}\right)\right)-a_{0}\right) / \alpha_{0}\right),} & \text { if } \alpha_{0}>0, \alpha_{1}=0, a_{1}<\mu_{1}^{\max }, \bar{\mu}_{0}\left(M_{1}^{1}\left(a_{1}\right)\right)>a_{0} \\ {\left[0, \bar{D}_{1}\right],} & \text { if } \alpha_{0} \geq 0, \alpha_{1}>0, \text { and case } 1 \text { of }(4.1) \text { holds } \\ \left.0, \hat{D}_{1}\right), & \text { if } \alpha_{0} \geq 0, \alpha_{1}>0, \text { and case } 2 \text { of }(4.1) \text { holds. }\end{cases}
$$


Proof. The function $F_{i}^{1}, i=1,2$, is defined if and only if

$$
D_{1}<\mu_{1}^{\max } \text { and } D_{0}<\bar{\mu}_{0}\left(M_{1}^{1}\left(D_{1}\right)\right) .
$$

If $\alpha_{0}=\alpha_{1}=0,(\mathrm{~A} .3)$ is equivalent to $a_{1}<\mu_{1}^{\max }$ and $a_{0}<\bar{\mu}_{0}\left(M_{1}^{1}\left(a_{1}\right)\right)$. Thus, $I_{1}=[0,+\infty)$ if this last condition holds, else $I_{1}=\emptyset$. If $\alpha_{0}>0$ and $\alpha_{1}=0$, (A.3) is equivalent to $a_{1}<\mu_{1}^{\max }$ and $\alpha_{0} D+a_{0}<\bar{\mu}_{0}\left(M_{1}^{1}\left(a_{1}\right)\right)$, that is, $I_{1}=\left[0,\left(\bar{\mu}_{0}\left(M_{1}^{1}\left(a_{1}\right)\right)-a_{0}\right) / \alpha_{0}\right)$. If $\alpha_{0} \geq 0$ and $\alpha_{1}>0$, straightforward calculation shows that

$$
\Phi_{j}^{\prime}(D)=\alpha_{1} \bar{\mu}_{0}^{\prime}\left(M_{1}^{j}\left(D_{1}\right)\right) M_{1}^{j \prime}\left(D_{1}\right)-\alpha_{0} .
$$

Recall that the function $M_{1}^{1}$ is increasing. From Hypothesis 2.5, one has $\Phi_{1}^{\prime}(D)<0$ for all $D \in \bar{I}_{1}$. Therefore, $\Phi_{1}(D)>0$ for all $D \in\left[0, \bar{D}_{1}\right]$ since $\Phi_{1}\left(\bar{D}_{1}\right)>0$ when case 1 of (4.1) holds and $\Phi_{1}(D)>0$ for all $D \in\left[0, \hat{D}_{1}\right)$ since $\Phi_{1}\left(\bar{D}_{1}\right) \leq 0$ when case 2 of (4.1) holds (see Figures 8 and 9 ).

When the growth functions are given by (C.1) (as we will show in Proposition C.1), the equation $\Phi_{2}(D)=0$ has at most three solutions in the case 1 of (4.1) and two solutions in the case 2 of (4.1). For simplicity, we determine in this particular case the definition domain $I_{2}$ of the function $F_{i}^{2}, i=1,2$. The general case can be treated similarly, without added difficulty.

Proposition A.3. The function $F_{i}^{2}$ is defined on

$$
I_{2}= \begin{cases}{[0,+\infty),} & \text { if } \alpha_{0}=0, \alpha_{1}=0, \bar{\mu}_{0}\left(M_{1}^{2}\left(a_{1}\right)\right)>a_{0}, \\ {\left[0,\left(\bar{\mu}_{0}\left(M_{1}^{2}\left(a_{1}\right)\right)-a_{0}\right) / \alpha_{0}\right),} & \text { if } \alpha_{0}>0, \alpha_{1}=0, \bar{\mu}_{0}\left(M_{1}^{2}\left(a_{1}\right)\right)>a_{0}, \\ {\left[0, \bar{D}_{1}\right],} & \text { if } \alpha_{0}=0, \alpha_{1}>0, \Phi_{2}\left(\bar{D}_{1}\right)>0, \bar{\mu}_{0}\left(M_{1}^{2}\left(a_{1}\right)\right)>a_{0}, \\ \left(\widetilde{D}_{1}, \bar{D}_{1}\right], & \text { if } \alpha_{0}=0, \alpha_{1}>0, \Phi_{2}\left(\bar{D}_{1}\right)>0, \bar{\mu}_{0}\left(M_{1}^{2}\left(a_{1}\right)\right) \leq a_{0},\end{cases}
$$

otherwise, when $\alpha_{0}>0$ and $\alpha_{1}>0$,

$$
I_{2}= \begin{cases}{\left[0, \bar{D}_{1}\right],} & \text { if case } 1 \text { of }(4.1) \text { holds and } n=0, \\ \left(\widetilde{D}_{1}, \bar{D}_{1}\right], & \text { if case } 1 \text { of }(4.1) \text { holds and } n=1, \\ {\left[0, \widetilde{D}_{2}\right) \cup\left(\widetilde{D}_{1}, \bar{D}_{1}\right],} & \text { if case } 1 \text { of }(4.1) \text { holds and } n=2, \\ \left(\widetilde{D}_{3}, \widetilde{D}_{2}\right) \cup\left(\widetilde{D}_{1}, \bar{D}_{1}\right], & \text { if case } 1 \text { of }(4.1) \text { holds and } n=3, \\ {\left[0, \widetilde{D}_{1}\right),} & \text { if case 2 of }(4.1) \text { holds and } n=1, \\ \left(\widetilde{D}_{2}, \widetilde{D}_{1}\right) & \text { if case } 2 \text { of }(4.1) \text { holds and } n=2,\end{cases}
$$

where $\widetilde{D}_{i}, i=1, \ldots, n$, are the solutions of the equation $\Phi_{2}(D)=0$ and $n$ denotes the number of solutions such that $\widetilde{D}_{i}>\widetilde{D}_{j}$, for all $i<j$. Note that the function $F_{i}^{2}$ is not defined for $D=0$ in the particular case $a_{1}=0$.

Proof. The function $F_{i}^{2}, i=1,2$, is defined if and only if

$$
0<D_{1}<\mu_{1}^{\max } \text { and } \Phi_{2}(D)>0 .
$$

If $\alpha_{1}=0$, similar arguments as the proof of $I_{1}$ imply that of $I_{2}$. If $\alpha_{0}=0$ and $\alpha_{1}>0$, then $\Phi_{2}^{\prime}(D)$ is positive for all $D \in \bar{I}_{2}$, using (A.4), Hypothesis 2.5 and the function $M_{1}^{2}$ is decreasing. Hence, $\Phi_{2}(D)>0$ is positive if and only if $\Phi_{2}\left(\bar{D}_{1}\right)>0$. In this case, $\Phi_{2}(D)>0$ for all $D \in\left[0, \bar{D}_{1}\right]$ if $\Phi_{2}(0)>0$, that is, $\bar{\mu}_{0}\left(M_{1}^{2}\left(a_{1}\right)\right)>a_{0}$. Otherwise, $\Phi_{2}(D)>0$ for all $D \in\left(\widetilde{D}_{1}, \bar{D}_{1}\right]$ where $\widetilde{D}_{1}$ is the unique solution of $\Phi_{2}(D)=0$.

Let $\alpha_{0}>0$ and $\alpha_{1}>0$. From (A.4), the sign of $\Phi_{2}^{\prime}(D)$ can change at $D \in \bar{I}_{2}$, that is, the function $\Phi_{2}$ can be nonmonotonic on $\bar{I}_{2}$ (see Figures 8 and $9(\mathrm{~b}-\mathrm{d})$ ). When case 1 holds and $n=0$, we have $\Phi_{2}\left(\bar{D}_{1}\right)>0$ and the equation $\Phi_{2}(D)=0$ has no solution. Consequently, $\Phi_{2}(D)>0$ for all $D \in\left[0, \bar{D}_{1}\right]$. Hence, the function $F_{i}^{2}$ is defined on $I_{2}=\bar{I}_{2}$ where $\bar{I}_{2}=\left[0, \bar{D}_{1}\right]$ when $a_{1}>0$ and $\bar{I}_{2}=\left(0, \bar{D}_{1}\right]$ when $a_{1}=0$. When case 1 holds and $n=1$, the equation $\Phi_{2}(D)=0$ has a unique solution $\widetilde{D}_{1} \in\left[0, \bar{D}_{1}\right]$. Thus, the function $F_{i}^{2}$ is defined on $\left(\widetilde{D}_{1}, \bar{D}_{1}\right]$ since $\Phi_{2}(D)>0$ for all $D \in\left(\widetilde{D}_{1}, \bar{D}_{1}\right]$. The other cases can be treated similarly (see Figure $8(\mathrm{~b}-\mathrm{d})$ ). 
The following result describes the properties of the functions $F_{0}$ and $F_{i}^{j}, i, j=1,2$, when $s_{1}^{i n}$ is fixed.

Proposition A.4. Let $s_{1}^{\text {in }} \geq 0$. We have $I_{2} \subset I_{1}, F_{i}^{1}(D) \leq F_{i}^{2}(D), i=1,2$, for all $D \in I_{2}$ and

$$
\lim _{D \rightarrow \bar{D}_{0}^{-}} F_{0}\left(D, s_{1}^{i n}\right)=\lim _{D \rightarrow \hat{D}_{1}^{-}} F_{i}^{1}(D)=\lim _{D \rightarrow \widetilde{D}_{j}^{-}} F_{i}^{2}(D)=+\infty, \quad j=1, \ldots, n .
$$

When case 1 of (4.1) holds, we have $F_{i}^{1}\left(\bar{D}_{1}\right)=F_{i}^{2}\left(\bar{D}_{1}\right), i=1,2$.

Proof. Using Hypothesis 2.5, given that $M_{1}^{1}\left(D_{1}\right) \leq M_{1}^{2}\left(D_{1}\right)$, for all $D \in \bar{I}_{2}$, we can write

$$
\Phi_{1}(D)=\bar{\mu}_{0}\left(M_{1}^{1}\left(D_{1}\right)\right)-D_{0} \geq \bar{\mu}_{0}\left(M_{1}^{2}\left(D_{1}\right)\right)-D_{0}=\Phi_{2}(D) .
$$

If $D \in I_{2}$, that is, $D \in \bar{I}_{2}$ such that $\Phi_{2}(D)>0$, then $D \in \bar{I}_{1}$ and $\Phi_{1}(D) \geq \Phi_{2}(D)>0$, that is, $D \in I_{1}$. Thus, $I_{2} \subset I_{1}$. Under Hypothesis 2.3 and the definition of $M_{0}$ in Table 3 , we have for all $y \in\left[0, \bar{\mu}_{0}\left(s_{1}\right)\right)$ and $s_{1} \geq 0$,

$$
\frac{\partial M_{0}}{\partial s_{1}}\left(y, s_{1}\right)=-\left[\frac{\partial \mu_{0}}{\partial s_{1}}\left(M_{0}\left(y, s_{1}\right), s_{1}\right)\right]\left[\frac{\partial \mu_{0}}{\partial s_{0}}\left(M_{0}\left(y, s_{1}\right), s_{1}\right)\right]^{-1}>0 .
$$

From the definition of $F_{1}^{j}$ in Table 3, it follows that

$$
F_{1}^{1}(D)=M_{0}\left(D_{0}, M_{1}^{1}\left(D_{1}\right)\right) \leq M_{0}\left(D_{0}, M_{1}^{2}\left(D_{1}\right)\right)=F_{1}^{2}(D) \text {, for all } D \in I_{2} .
$$

Similarly, $F_{2}^{1}(D) \leq F_{2}^{2}(D)$, for all $D \in I_{2}$. From the definitions of $F_{0}$ and $\bar{D}_{0}\left(s_{1}^{i n}\right)$ in Tables 3 and 9 , we then obtain

$$
F_{0}\left(\bar{D}_{0}\left(s_{1}^{i n}\right), s_{1}^{i n}\right)=M_{0}\left(\alpha_{0} \bar{D}_{0}\left(s_{1}^{i n}\right)+a_{0}, s_{1}^{i n}\right)=M_{0}\left(\bar{\mu}_{0}\left(s_{1}^{i n}\right), s_{1}^{i n}\right)=+\infty .
$$

When case 1 of (4.1) holds, the function $F_{i}^{j}, i, j=1,2$, is defined for $D=\bar{D}_{1}$. Using Hypothesis 2.4 and the definition of $\bar{D}_{1}$ in Table 9, it follows that

$$
M_{1}^{1}\left(\alpha_{1} \bar{D}_{1}+a_{1}\right)=M_{1}^{1}\left(\mu_{1}^{\max }\right)=M_{1}^{2}\left(\mu_{1}^{\max }\right)=s_{1}^{\max } .
$$

Consequently,

$$
F_{1}^{1}\left(\bar{D}_{1}\right)=M_{0}\left(\alpha_{0} \bar{D}_{1}+a_{0}, s_{1}^{\max }\right)=F_{1}^{2}\left(\bar{D}_{1}\right), F_{2}^{1}\left(\bar{D}_{1}\right)=M_{0}\left(\alpha_{0} \bar{D}_{1}+a_{0}, s_{1}^{\max }\right)+s_{1}^{\max }=F_{2}^{2}\left(\bar{D}_{1}\right) .
$$

The last limit follows similarly.

\section{Appendix B. Proofs.}

Proof of Proposition 2.6. Since the vector field defined by system (2.1) is $\mathcal{C}^{1}$, the uniqueness of solution to initial value problems holds. From $(2.1), x_{i}\left(t_{0}\right)=0$, for any $t_{0} \geqslant 0$ implies $\dot{x}_{i}\left(t_{0}\right)=0$, $i=0,1$. If $x_{i}(0)=0$, then $x_{i}(t)=0$ for all $t$ as the boundary face $x_{i} \equiv 0$ is invariant in the vector field $C^{1}$ by (2.1). If $x_{i}(0)>0$, then $x_{i}(t)>0$ for all $t$ as $x_{i} \equiv 0$ cannot be reached in finite time by trajectories for which $x_{i}(0)>0$ by the uniqueness of solutions. On the other hand, we have

$$
s_{0}\left(t_{0}\right)=0 \text { for any } t_{0} \geqslant 0 \Rightarrow \dot{s}_{0}\left(t_{0}\right)=D s_{0}^{i n} .
$$

If $\dot{s}_{0}\left(t_{0}\right)=0$, then $s_{0}(t) \geqslant 0$ for all $t$, using arguments similar to case $x_{i}$. However, if $\dot{s}_{0}\left(t_{0}\right)>0$, then $s_{0}(t) \geqslant 0$ for all $t$. In fact, assume that $s_{0}(0) \geq 0$ and that it exists $t_{0}>0$, such that $s_{0}\left(t_{0}\right)=0$ and $s_{0}(t)>0$, for $t \in\left(0, t_{0}\right)$. Then $\dot{s}_{0}\left(t_{0}\right) \leq 0$ which contradicts $\dot{s}_{0}\left(t_{0}\right)>0$. Finally, we have

$$
s_{1}\left(t_{0}\right)=0 \text { for any } t_{0} \geqslant 0 \Rightarrow \dot{s}_{1}\left(t_{0}\right)=D s_{1}^{i n}+\mu_{0}\left(s_{0}\left(t_{0}\right), 0\right) x_{0}\left(t_{0}\right) .
$$


Similarly to case $s_{0}$, no trajectory can leave the positive octant $\mathbb{R}_{+}^{4}$ by crossing the boundary face $s_{1}=0$ since $s_{0} \geq 0$ and $x_{0} \geq 0$. Therefore, all solutions of (2.1) remain nonnegative. Let $z=2 s_{0}+x_{0}+s_{1}+x_{1}$. From (2.1), it follows that

$$
\dot{z}=D\left(2 s_{0}^{i n}+s_{1}^{i n}-2 s_{0}-s_{1}\right)-D_{0} x_{0}-D_{1} x_{1} \leq D_{\min }\left(\frac{D}{D_{\min }}\left(2 s_{0}^{i n}+s_{1}^{i n}\right)-z\right) .
$$

By applying Gronwall's lemma, we obtain

$$
z(t) \leq \frac{D}{D_{\min }}\left(2 s_{0}^{i n}+s_{1}^{i n}\right)+\left(z(0)-\frac{D}{D_{\min }}\left(2 s_{0}^{i n}+s_{1}^{i n}\right)\right) e^{-D_{\min } t}, \quad \text { for all } t \geq 0
$$

We deduce that

$$
z(t) \leq \max \left(z(0), \frac{D}{D_{\min }}\left(2 s_{0}^{i n}+s_{1}^{i n}\right)\right), \text { for all } t \geq 0 .
$$

Consequently, the solutions of (2.1) are bounded for all $t \geq 0$. Inequality (B.1) implies that the set $\Omega$ is positively invariant and is a global attractor for (2.1).

Proof of Proposition 3.1. The steady states of (2.1) are the solutions of the following set of equation

$$
\begin{aligned}
D\left(s_{0}^{i n}-s_{0}\right)-\mu_{0}\left(s_{0}, s_{1}\right) x_{0} & =0 \\
\left(\mu_{0}\left(s_{0}, s_{1}\right)-D_{0}\right) x_{0} & =0 \\
D\left(s_{1}^{i n}-s_{1}\right)+\mu_{0}\left(s_{0}, s_{1}\right) x_{0}-\mu_{1}\left(s_{1}\right) x_{1} & =0 \\
\left(\mu_{1}\left(s_{1}\right)-D_{1}\right) x_{1} & =0 .
\end{aligned}
$$

Using (B.2)+(B.3) and (B.4)-(B.3)+(B.5), we obtain the set of equations

$$
\left\{\begin{array}{l}
D\left(s_{0}^{\text {in }}-s_{0}\right)-D_{0} x_{0}=0 \\
D\left(s_{1}^{i n}-s_{1}\right)+D_{0} x_{0}-D_{1} x_{1}=0 .
\end{array}\right.
$$

By solving (B.6), we obtain $x_{0}$ and $x_{1}$ with respect to $s_{0}$ and $s_{1}$ :

$$
\begin{aligned}
& x_{0}=\frac{D}{D_{0}}\left(s_{0}^{i n}-s_{0}\right), \\
& x_{1}=\frac{D}{D_{1}}\left(s_{0}^{i n}-s_{0}+s_{1}^{i n}-s_{1}\right) .
\end{aligned}
$$

We can also solve (B.6) and obtain $s_{0}$ and $s_{1}$ with respect to $x_{0}$ and $x_{1}$ :

$$
\begin{aligned}
& s_{0}=s_{0}^{i n}-\frac{D_{0}}{D} x_{0}, \\
& s_{1}=s_{1}^{i n}+\frac{D_{0}}{D} x_{0}-\frac{D_{1}}{D} x_{1} .
\end{aligned}
$$

For $\mathrm{SS}_{0}$, one has $x_{0}=x_{1}=0$. Hence, (B.9) and (B.10) result in $s_{0}=s_{0}^{i n}$ and $s_{1}=s_{1}^{i n}$. Thus, $\mathrm{SS}_{0}$ always exists.

For $\mathrm{SS}_{1}$, one has $x_{0}>0, x_{1}=0$. Hence, (B.7) results in

$$
x_{0}=\frac{D}{D_{0}}\left(s_{0}^{i n}-s_{0}\right) .
$$

Using (B.8) and $x_{1}=0$, it follows that

$$
s_{1}=s_{1}^{i n}+s_{0}^{i n}-s_{0}
$$

Since $x_{0}>0,($ B.3) results in

$$
\psi\left(s_{0}\right):=\mu_{0}\left(s_{0}, s_{1}^{i n}+s_{0}^{i n}-s_{0}\right)=D_{0} .
$$

Thus, $\mathrm{SS}_{1}$ exists if and only if equation (B.11) has a nonnegative solution and the $s_{1}$ and $x_{0}$-components are positive. This condition is equivalent to say that $0 \leq s_{0}<s_{0}^{i n}$. From Hypotheses 2.1 and 2.3, we 
see that the function $s_{0} \mapsto \psi\left(s_{0}\right)$ is strictly increasing from 0 for $s_{0}=0$ to $\mu_{0}\left(s_{0}^{i n}, s_{1}^{i n}\right)$ for $s_{0}=s_{0}^{i n}$. Therefore, there exists a solution of equation (B.11) in $\left[0, s_{0}^{i n}\right)$ if and only if

$$
\mu_{0}\left(s_{0}^{i n}, s_{1}^{i n}\right)>D_{0} .
$$

If such a solution exists, then it is unique. Under Hypothesis 2.3 and the definition of $M_{0}$ in Table 3 , we have for all $y \in\left[0, \bar{\mu}_{0}\left(s_{1}\right)\right)$ and $s_{1} \geq 0$,

$$
\frac{\partial M_{0}}{\partial y}\left(y, s_{1}\right)=\left[\frac{\partial \mu_{0}}{\partial s_{0}}\left(M_{0}\left(y, s_{1}\right), s_{1}\right)\right]^{-1}>0 .
$$

Using (B.13), condition (B.12) is equivalent to the existence condition of $\mathrm{SS}_{1}$ in Table 5.

For $\mathrm{SS}_{2}^{j}, j=1,2$, one has $x_{0}>0$ and $x_{1}>0$. Hence, (B.7) and (B.8) result in

$$
x_{0}=\frac{D}{D_{0}}\left(s_{0}^{i n}-s_{0}\right), \quad x_{1}=\frac{D}{D_{1}}\left(s_{0}^{i n}+s_{1}^{i n}-s_{0}-s_{1}\right) .
$$

Moreover, (B.3) and (B.5) result in $\mu_{0}\left(s_{0}, s_{1}\right)=D_{0}$ and $\mu_{1}\left(s_{1}\right)=D_{1}$. Using the definitions of $M_{0}$ and $M_{1}^{j}$ in Table 3, we obtain

$$
s_{0}=M_{0}\left(D_{0}, s_{1}\right), \quad s_{1}=M_{1}^{j}\left(D_{1}\right),
$$

where the function $M_{1}^{j}\left(D_{1}\right)$ is defined for all $D \in \bar{I}_{j}$. From Hypotheses 2.1, 2.3, and 2.5, the function $s_{0} \mapsto \mu_{0}\left(s_{0}, M_{1}^{j}\left(D_{1}\right)\right)$ is strictly increasing from 0 for $s_{0}=0$ to $\bar{\mu}_{0}\left(M_{1}^{j}\left(D_{1}\right)\right)$ when $s_{0}$ tends towards infinity. Using the definitions of $\Phi_{j}(D)$ and $I_{j}$ in Table 9 , equation $\mu_{0}\left(s_{0}, M_{1}^{j}\left(D_{1}\right)\right)=D_{0}$ has a solution $s_{0} \geq 0$ if and only if

$$
\Phi_{j}(D):=\bar{\mu}_{0}\left(M_{1}^{j}\left(D_{1}\right)\right)-D_{0}>0, \quad \text { with } \quad D \in \bar{I}_{j},
$$

or equivalently $D \in I_{j}$. Thus, $\mathrm{SS}_{2}^{j}$ exists if and only if $s_{0}^{i n}+s_{1}^{i n}>s_{0}+s_{1}$ and $s_{0}^{i n}>s_{0}$, that is, the existence condition in Table 5 is satisfied with $D \in I_{j}$.

For $\mathrm{SS}_{3}^{j}, j=1,2$, one has $x_{0}=0$ and $x_{1}>0$. Hence, (B.8) and (B.9) result in

$$
s_{0}=s_{0}^{i n}, \quad x_{1}=\frac{D}{D_{1}}\left(s_{1}^{i n}-s_{1}\right) .
$$

Since $x_{1}>0$, (B.5) results in $\mu_{1}\left(s_{1}\right)=D_{1}$. Using the definitions of $M_{1}^{j}$ in Table 3 , we obtain

$$
s_{1}=M_{1}^{j}\left(D_{1}\right), \quad \text { where } \quad D \in \bar{I}_{j} .
$$

Thus, we conclude that $\mathrm{SS}_{3}^{j}$ exists if and only if $s_{1}^{\text {in }}>M_{1}^{j}\left(D_{1}\right)$.

In what follows, we determine the local asymptotic stability of each steady state of (2.1). Let $J$ be the Jacobian matrix of (2.1) at a steady state $\left(s_{0}, x_{0}, s_{1}, x_{1}\right)$, that is given by

$$
J=\left[\begin{array}{cccc}
-D-E x_{0} & -\mu_{0} & F x_{0} & 0 \\
E x_{0} & \mu_{0}-D_{0} & -F x_{0} & 0 \\
E x_{0} & \mu_{0} & -D-F x_{0}-\mu_{1}^{\prime} x_{1} & -\mu_{1} \\
0 & 0 & \mu_{1}^{\prime} x_{1} & \mu_{1}-D_{1}
\end{array}\right]
$$

where

$$
E=\frac{\partial \mu_{0}}{\partial s_{0}}\left(s_{0}, s_{1}\right)>0, \quad F=-\frac{\partial \mu_{0}}{\partial s_{1}}\left(s_{0}, s_{1}\right)>0 .
$$

For $\mathrm{SS}_{0}$, the characteristic polynomial is $P_{0}(\lambda)=\left(\lambda-\lambda_{1}\right)\left(\lambda-\lambda_{2}\right)(\lambda+D)^{2}$, where

$$
\lambda_{1}=\mu_{0}\left(s_{0}^{i n}, s_{1}^{i n}\right)-D_{0}, \quad \lambda_{2}=\mu_{1}\left(s_{1}^{\text {in }}\right)-D_{1} .
$$

Thus, $\mathrm{SS}_{0}$ is LES if and only if

$$
\mu_{0}\left(s_{0}^{i n}, s_{1}^{i n}\right)<D_{0} \quad \text { and } \quad \mu_{1}\left(s_{1}^{i n}\right)<D_{1} .
$$


If $D_{0} \geq \bar{\mu}_{0}\left(s_{1}^{i n}\right)$, that is, $D \notin I_{0}$, then the first condition of (B.15) is satisfied. If $D \in I_{0}$, using (B.13) and the definition of $M_{0}$ in Table 3, it follows that the first condition of (B.15) is equivalent to

$$
s_{0}^{i n}<M_{0}\left(D_{0}, s_{1}^{i n}\right)=F_{0}\left(D, s_{1}^{i n}\right) .
$$

If $D_{1}>\mu_{1}^{\max }$, that is, $D \notin \bar{I}_{1}$, then the second condition of (B.15) is satisfied. If $D \in \bar{I}_{j}, j=1,2$, using the definition of $M_{1}^{j}$ in Table 3, it follows that the second condition of (B.15) is equivalent to

$$
s_{1}^{i n}<M_{1}^{1}\left(D_{1}\right) \quad \text { with } \quad D \in \bar{I}_{1} \quad \text { or } \quad s_{1}^{i n}>M_{1}^{2}\left(D_{1}\right) \quad \text { with } \quad D \in \bar{I}_{2} .
$$

At $\mathrm{SS}_{1}$, the Jacobian matrix is given by

$$
J=\left[\begin{array}{cccc}
-D-E x_{0} & -D_{0} & F x_{0} & 0 \\
E x_{0} & 0 & -F x_{0} & 0 \\
E x_{0} & D_{0} & -D-F x_{0} & -\mu_{1} \\
0 & 0 & 0 & \mu_{1}-D_{1}
\end{array}\right] .
$$

Denote $C_{i}$ and $L_{i}$ the columns and lines of the matrix $J-\lambda I$. The replacements of $L_{1}$ by $L_{1}+L_{3}$ and then $C_{3}$ by $C_{3}-C_{1}$ preserve the determinant and lead to the following characteristic polynomial

$$
P_{1}(\lambda)=\left(\lambda-\lambda_{1}\right)\left(\lambda-\lambda_{1}\right)\left(\lambda^{2}+c_{1} \lambda+c_{2}\right),
$$

where $\lambda_{1}=\mu_{1}\left(s_{0}^{i n}+s_{1}^{i n}-s_{0}\right)-D_{1}, \lambda_{2}=-D, c_{1}=D+(E+F) x_{0}$ and $c_{2}=D_{0}(E+F) x_{0}$. Since $c_{1}>0$ and $c_{2}>0$, the real parts of the roots of the quadratic factor are negative. Therefore, $\mathrm{SS}_{1}$ is LES if and only if $\lambda_{1}<0$, that is,

$$
\mu_{1}\left(s_{0}^{i n}+s_{1}^{i n}-s_{0}\right)<D_{1} .
$$

If $D_{1}>\mu_{1}^{\max }$, that is, $D \notin \bar{I}_{1}$, then condition (B.17) is satisfied. If $D_{1} \leq \mu_{1}^{\max }$, that is, $D \in \bar{I}_{1}$, then condition (B.17) is equivalent to

$$
s_{0}>s_{0}^{i n}+s_{1}^{i n}-M_{1}^{1}\left(D_{1}\right) \quad \text { or } \quad s_{0}<s_{0}^{i n}+s_{1}^{i n}-M_{1}^{2}\left(D_{1}\right) .
$$

Since the function $s_{0} \mapsto \psi\left(s_{0}\right)=\mu_{0}\left(s_{0}, s_{0}^{i n}+s_{1}^{i n}-s_{0}\right)$ is increasing, (B.18) is equivalent to

$$
\psi\left(s_{0}\right)>\psi\left(s_{0}^{i n}+s_{1}^{i n}-M_{1}^{1}\left(D_{1}\right)\right) \quad \text { or } \quad \psi\left(s_{0}\right)<\psi\left(s_{0}^{i n}+s_{1}^{i n}-M_{1}^{2}\left(D_{1}\right)\right) .
$$

At $\mathrm{SS}_{1}$, one has $\psi\left(s_{0}\right)=D_{0}$. Thus, condition (B.18) is equivalent to

$$
D_{0}>\mu_{0}\left(s_{0}^{i n}+s_{1}^{i n}-M_{1}^{1}\left(D_{1}\right), M_{1}^{1}\left(D_{1}\right)\right) \quad \text { or } \quad D_{0}<\mu_{0}\left(s_{0}^{i n}+s_{1}^{i n}-M_{1}^{2}\left(D_{1}\right), M_{1}^{2}\left(D_{1}\right)\right) .
$$

If $D \in \bar{I}_{1} \backslash I_{1}$, that is, $\Phi_{1}(D)<0$ (or equivalently $\left.\bar{\mu}_{0}\left(M_{1}^{1}\left(D_{1}\right)\right)<D_{0}\right)$ then the first condition of (B.19) is satisfied. If $D \in \bar{I}_{1} \backslash I_{2}$, that is, $\bar{\mu}_{0}\left(M_{1}^{2}\left(D_{1}\right)\right)<D_{0}$, then the second condition of (B.19) is not satisfied. If $D \in I_{j}, j=1,2$, then condition (B.19) is equivalent to

$$
s_{0}^{i n}+s_{1}^{i n}<M_{0}\left(D_{0}, M_{1}^{1}\left(D_{1}\right)\right)+M_{1}^{1}\left(D_{1}\right) \quad \text { or } \quad s_{0}^{i n}+s_{1}^{i n}>M_{0}\left(D_{0}, M_{1}^{2}\left(D_{1}\right)\right)+M_{1}^{2}\left(D_{1}\right)
$$

because the function $M_{0}\left(\cdot, M_{1}^{1}\left(D_{1}\right)\right)$ is increasing. These conditions are the same as those in Table 5 .

For $\mathrm{SS}_{2}^{j}, j=1,2$, the characteristic polynomial is $P_{2}(\lambda)=\lambda^{4}+c_{1} \lambda^{3}+c_{2} \lambda^{2}+c_{3} \lambda+c_{4}$, where

$$
\begin{aligned}
& c_{1}=G_{j} x_{1}+H x_{0}+2 D, \quad c_{2}=E G_{j} x_{0} x_{1}+\left(D+D_{0}\right) H x_{0}+\left(D+D_{1}\right) G_{j} x_{1}+D^{2}, \\
& c_{3}=\left(D_{0}+D_{1}\right) E G_{j} x_{0} x_{1}+D D_{0} H x_{0}+D D_{1} G_{j} x_{1}, \quad c_{4}=D_{0} D_{1} E G_{j} x_{0} x_{1},
\end{aligned}
$$

with $H=E+F$ and $G_{1}:=\mu_{1}^{\prime}\left(M_{1}^{1}\left(D_{1}\right)\right)>0$ since $M_{1}^{1}\left(D_{1}\right)<s_{1}^{\max }$ and $G_{2}:=\mu_{1}^{\prime}\left(M_{1}^{2}\left(D_{1}\right)\right)<0$ since $M_{1}^{j}\left(D_{1}\right)>s_{1}^{\max }$. Note that $G_{1}=G_{2}=0$, when $D=\bar{D}_{1}$ where $\mathrm{SS}_{2}^{1}=\mathrm{SS}_{2}^{2}$. Using the Liénard-Chipart stability criteria (see Gantmacher [20], Theorem 11), $\mathrm{SS}_{2}^{j}$ is LES if and only if

$c_{i}>0, \quad i=1,3,4, \quad$ and $\quad c_{1} c_{2} c_{3}-c_{1}^{2} c_{4}-c_{3}^{2}>0$. 
Hence, $\mathrm{SS}_{2}^{2}$ is unstable as long as it exists with $D \neq \bar{D}_{1}$ because the condition $c_{4}>0$ in (B.20) is unfulfilled as $G_{2}<0$. For $\mathrm{SS}_{2}^{1}, c_{i}>0$, for all $i=1,3,4$, as $E, F, H$ and $G_{1}$ are positive. Following [40], where the particular case $\alpha_{0}=\alpha_{1}=1$ was considered, we obtain

$$
c_{1} c_{2} c_{3}-c_{1}^{2} c_{4}-c_{3}^{2}=\gamma_{5} D^{5}+\gamma_{4} D^{4}+\gamma_{3} D^{3}+\gamma_{2} D^{2}+\gamma_{1} D+\gamma_{0},
$$

where the coefficients $\gamma_{j}, j=0, \ldots, 5$, can be written as follows:

$$
\begin{aligned}
& \gamma_{5}=2\left(\alpha_{0} H x_{0}+\alpha_{1} G_{1} x_{1}\right), \\
& \gamma_{4}=2\left[\alpha_{0}\left(1-\alpha_{1}\right)+\alpha_{1}\left(1-\alpha_{0}\right)\right] E G_{1} x_{0} x_{1}+\left(\left(3+\alpha_{0}\right) H x_{0}+\left(3+\alpha_{1}\right) G_{1} x_{1}\right)\left(\alpha_{0} H x_{0}+\alpha_{1} G_{1} x_{1}\right) \\
& +2 a_{0} H x_{0}+2 a_{1} G_{1} x_{1} \\
& \left.\gamma_{3}=\left[2\left(\alpha_{1} a_{0}+\alpha_{0} a_{1}\right) H+\left(a_{0}\left(5-4 \alpha_{1}\right)+a_{1}\left(5-4 \alpha_{0}\right)\right) E+3\left(a_{0}+a_{1}\right) F\right)\right] G_{1} x_{0} x_{1} \\
& +\left[\alpha_{1}\left(\alpha_{0}+1\right) H+\left(\left(7-3 \alpha_{1}\right) \alpha_{0}+3 \alpha_{1}+\alpha_{0}^{2}\right) E+\alpha_{0}\left(\alpha_{0}+\alpha_{1}+2\right) F\right] H G_{1} x_{0}^{2} x_{1} \\
& +\left[\alpha_{0}\left(\alpha_{1}+1\right) H+\left(\left(7-3 \alpha_{0}\right) \alpha_{1}+3 \alpha_{0}+\alpha_{1}^{2}\right) E+\alpha_{1}\left(\alpha_{0}+\alpha_{1}+2\right) F\right] G_{1}^{2} x_{0} x_{1}^{2} \\
& +\left(3+2 \alpha_{0}\right) a_{0} H^{2} x_{0}^{2}+\left(3+2 \alpha_{1}\right) a_{1} G_{1}^{2} x_{1}^{2}+\alpha_{0}\left(\alpha_{0}+1\right) H^{3} x_{0}^{3}+\alpha_{1}\left(\alpha_{1}+1\right) G_{1}^{3} x_{1}^{3} \text {, } \\
& \gamma_{2}=\left[a_{0}\left(\left(2 \alpha_{0}+\alpha_{1}+2\right) H+\left(5-3 \alpha_{1}\right) E+\alpha_{1} F\right)+a_{1}\left(\left(\alpha_{0}+1\right) H+3\left(1-\alpha_{0}\right) E+\alpha_{0} F\right)\right] H G_{1} x_{0}^{2} x_{1} \\
& +\left[a_{0}\left(\left(\alpha_{1}+1\right) H+3\left(1-\alpha_{1}\right) E+\alpha_{1} F\right)+a_{1}\left(2\left(\alpha_{0}+\alpha_{1}+1\right) H+\left(5-4 \alpha_{0}\right) E\right)\right] G_{1}^{2} x_{0} x_{1}^{2} \\
& +\left[\left(\left(3-2 \alpha_{1}\right) \alpha_{0}+\alpha_{1}\right) H+2\left(\alpha_{0}+2 \alpha_{1}\right) E+\left(\left(\alpha_{0}+\alpha_{1}\right)^{2}+2 \alpha_{1}\right) F\right] E G_{1}^{2} x_{0}^{2} x_{1}^{2} \\
& +\left(\alpha_{0}^{2}+2 \alpha_{0}+\alpha_{1}\right) E H^{2} G_{1} x_{0}^{3} x_{1}+\left(\alpha_{1}^{2}+2 \alpha_{1}+\alpha_{0}\right) E G_{1}^{3} x_{0} x_{1}^{3}+\left(1+2 \alpha_{0}\right) a_{0} H^{3} x_{0}^{3} \\
& +\left(1+2 \alpha_{1}\right) a_{1} G_{1}^{3} x_{1}^{3}+\left(a_{0} H x_{0}-a_{1} G_{1} x_{1}\right)^{2}+4 a_{0} a_{1} F G_{1} x_{0} x_{1}, \\
& \gamma_{1}=\left(H x_{0}+G_{1} x_{1}\right)\left(a_{0} H x_{0}-a_{1} G_{1} x_{1}\right)^{2}+4 a_{0} a_{1} F H G_{1} x_{0}^{2} x_{1}+4 a_{0} a_{1} F G_{1}^{2} x_{0} x_{1}^{2} \\
& +\left[a_{0}\left(\left(5-2 \alpha_{1}\right) E+\left(2 \alpha_{0}+3\right) F\right)+a_{1}\left(\left(5-2 \alpha_{0}\right) E+\left(2 \alpha_{1}+3\right) F\right)\right] E G_{1}^{2} x_{0}^{2} x_{1}^{2} \\
& +\left(2\left(\alpha_{0}+1\right) a_{0}+a_{1}\right) E H^{2} G_{1} x_{0}^{3} x_{1}+\left(a_{0}+2\left(\alpha_{1}+1\right) a_{1}\right) E G_{1}^{3} x_{0} x_{1}^{3} \\
& +\left(\alpha_{0}+\alpha_{1}\right)\left(H x_{0}+G_{1} x_{1}\right) E^{2} G_{1}^{2} x_{0}^{2} x_{1}^{2}, \\
& \gamma_{0}=\left(a_{0}+a_{1}\right)\left(H x_{0}+G_{1} x_{1}\right) E^{2} G_{1}^{2} x_{0}^{2} x_{1}^{2}+\left(a_{0}+a_{1}\right)^{2} E F G_{1}^{2} x_{0}^{2} x_{1}^{2}+\left(a_{0} H x_{0}-a_{1} G_{1} x_{1}\right)^{2} E G_{1} x_{0} x_{1} \text {. }
\end{aligned}
$$

Since $\alpha_{0}$ and $\alpha_{1}$ are in $[0,1]$, then $\gamma_{j}>0$ for $j=0, \ldots, 5$. Thus, the conditions of Liénard-Chipart stability criteria (B.20) are satisfied for $\mathrm{SS}_{2}^{1}$ which is LES as long as it exists with $D \neq \bar{D}_{1}$.

For $\mathrm{SS}_{3}^{j}, j=1,2$, the characteristic polynomial is

$$
P_{3}(\lambda)=\left(\lambda-\lambda_{1}\right)\left(\lambda-\lambda_{2}\right)\left(\lambda^{2}+c_{1} \lambda+c_{2}\right)
$$

where $\lambda_{1}=-D, \lambda_{2}=\mu_{0}\left(s_{0}^{i n}, M_{1}^{j}\left(D_{1}\right)\right)-D_{0}, c_{1}=D+G_{j} x_{1}$ and $c_{2}=D_{1} G_{j} x_{1}$. For $\mathrm{SS}_{3}^{2}, c_{2}<0$ since $G_{2}<0$. Therefore, the roots of the quadratic factor are real and have opposite signs. Consequently, if $\mathrm{SS}_{3}^{2}$ exists, it is unstable. For $\mathrm{SS}_{3}^{1}$, the real parts of the roots of the quadratic factor are negative as $G_{1}>0$ so that $c_{1}>0$ and $c_{2}>0$. Therefore, $\mathrm{SS}_{3}^{1}$ is LES if and only if

$$
\mu_{0}\left(s_{0}^{i n}, M_{1}^{1}\left(D_{1}\right)\right)<D_{0} \quad \Longleftrightarrow \quad s_{0}^{i n}<M_{0}\left(D_{0}, M_{1}^{1}\left(D_{1}\right)\right),
$$

since the function $M_{0}\left(\cdot, M_{1}^{1}\left(D_{1}\right)\right)$ is increasing.

Proof of Proposition 4.1. Assume that $s_{1}^{i n}<M_{1}^{1}\left(D_{1}\right)$. Using Table $5, \mathrm{SS}_{3}^{j}, j=1,2$ does not exist. In this case, we have $F_{1}^{j}(D)<F_{2}^{j}(D)-s_{1}^{\text {in }}$. If $\left(D, s_{0}^{i n}, s_{1}^{i n}\right) \in \mathcal{J}_{1}$, then $s_{0}^{i n}<F_{0}\left(D, s_{1}^{i n}\right)$. From Table $5, \mathrm{SS}_{0}$ is LES and $\mathrm{SS}_{1}$ does not exist. If $D \notin I_{j}$, then the existence condition of $\mathrm{SS}_{2}^{j}$ does not hold since $F_{1}^{j}(D)=F_{2}^{j}(D)=+\infty$. Let $D \in I_{j}$. Assume that the existence condition of $\mathrm{SS}_{2}^{j}$ holds. Then,

$$
M_{0}\left(D_{0}, M_{1}^{j}\left(D_{1}\right)\right)=F_{1}^{j}(D)<s_{0}^{i n}<F_{0}\left(D, s_{1}^{i n}\right)=M_{0}\left(D_{0}, s_{1}^{i n}\right) .
$$

Using (A.9), we obtain $M_{1}^{j}\left(D_{1}\right)<s_{1}^{\text {in }}$ which is the desired contradiction. If $\left(D, s_{0}^{i n}, s_{1}^{i n}\right) \in \mathcal{J}_{2}$, then $F_{0}\left(D, s_{1}^{i n}\right)<s_{0}^{i n}<F_{2}^{1}(D)-s_{1}^{i n}$. From Table $5, \mathrm{SS}_{0}$ is unstable and $\mathrm{SS}_{1}$ exists and is LES. If $D \notin I_{j}$, 
the existence condition of $\mathrm{SS}_{2}^{j}$ does not hold. Let $D \in I_{j}$. If $\mathrm{SS}_{2}^{j}$ exists, that is, $s_{0}^{i n}>F_{2}^{j}(D)-s_{1}^{i n}$. This is a contradiction for $j=1$. However, for $j=2$, one has $F_{2}^{1}(D)-s_{1}^{i n}<F_{2}^{2}(D)-s_{1}^{\text {in }}<s_{0}^{\text {in }}$ which is a contradiction. If $\left(D, s_{0}^{i n}, s_{1}^{i n}\right) \in \mathcal{J}_{3}$, then $F_{2}^{1}(D)-s_{1}^{\text {in }}<s_{0}^{\text {in }}<F_{2}^{2}(D)-s_{1}^{\text {in }}$. From Table $5, \mathrm{SS}_{2}^{1}$ exists and is LES while $\mathrm{SS}_{2}^{2}$ does not exist. Assume that $s_{0}^{\text {in }} \leq F_{0}\left(D, s_{1}^{\text {in }}\right)$. Hence,

$$
F_{1}^{1}(D)<F_{2}^{1}(D)-s_{1}^{i n}<F_{0}\left(D, s_{1}^{i n}\right), \quad \text { or (equivalently) } \quad M_{1}^{1}\left(D_{1}\right)<s_{1}^{i n},
$$

which is a contradiction, that is, $s_{0}^{i n}>F_{0}\left(D, s_{1}^{i n}\right)$. Therefore, $\mathrm{SS}_{0}$ exists and is unstable while $\mathrm{SS}_{1}$ exists and is LES, using Table 5. If $\left(D, s_{0}^{i n}, s_{1}^{i n}\right) \in \mathcal{J}_{4}$, then $s_{0}^{i n}>F_{2}^{2}(D)-s_{1}^{i n}>F_{2}^{1}(D)-s_{1}^{i n}>F_{1}^{1}(D)$. From Table 5, $\mathrm{SS}_{2}^{1}$ exists and is LES while $\mathrm{SS}_{2}^{2}$ exists and is unstable. Assume that $s_{0}^{\text {in }} \leq F_{0}\left(D, s_{1}^{\text {in }}\right)$. Hence,

$$
F_{1}^{1}(D)<F_{0}\left(D, s_{1}^{i n}\right), \quad \text { or (equivalently) } \quad M_{1}^{1}\left(D_{1}\right)<s_{1}^{i n},
$$

which is a contradiction. Thus, $s_{0}^{i n}>F_{0}\left(D, s_{1}^{i n}\right)$. Therefore, $\mathrm{SS}_{0}$ is unstable while $\mathrm{SS}_{1}$ is LES, using Table 5 .

Assume that $M_{1}^{1}\left(D_{1}\right)<s_{1}^{\text {in }}<M_{1}^{2}\left(D_{1}\right)$. Using Table $5, \mathrm{SS}_{0}$ is unstable, $\mathrm{SS}_{3}^{1}$ exists and $\mathrm{SS}_{3}^{2}$ does not exist. In this cas, we have $F_{1}^{1}(D)>F_{2}^{1}(D)-s_{1}^{i n}$ and $F_{1}^{2}(D)<F_{2}^{2}(D)-s_{1}^{i n}$. If $\left(D, s_{0}^{i n}, s_{1}^{i n}\right) \in \mathcal{J}_{5}$, then $s_{0}^{\text {in }}>F_{2}^{2}(D)-s_{1}^{\text {in }}>F_{1}^{2}(D)>F_{1}^{1}(D)$. Assume that $s_{0}^{\text {in }} \leq F_{0}\left(D, s_{1}^{\text {in }}\right)$. Hence,

$$
F_{1}^{2}(D)<F_{0}\left(D, s_{1}^{i n}\right), \quad \text { or (equivalently) } \quad M_{1}^{2}\left(D_{1}\right)<s_{1}^{i n}
$$

which is a contradiction. Thus, $s_{0}^{i n}>F_{0}\left(D, s_{1}^{i n}\right)$. From Table $5, \mathrm{SS}_{1}$ and $\mathrm{SS}_{2}^{1}$ exist and are LES while $\mathrm{SS}_{2}^{2}$ exists and is unstable. If $\left(D, s_{0}^{i n}, s_{1}^{i n}\right) \in \mathcal{J}_{6}$, then

$$
F_{2}^{1}(D)-s_{1}^{i n}<F_{1}^{1}(D)<F_{0}\left(D, s_{1}^{i n}\right)<s_{0}^{i n}<F_{2}^{2}(D)-s_{1}^{i n}
$$

because $M_{1}^{1}\left(D_{1}\right)<s_{1}^{\text {in }}$. Using Table $5, \mathrm{SS}_{1}$ and $\mathrm{SS}_{3}^{1}$ exist and are unstable, $\mathrm{SS}_{2}^{1}$ exists and is $\mathrm{LES}_{\mathrm{SS}}^{2}$ does not exist. If $\left(D, s_{0}^{i n}, s_{1}^{\text {in }}\right) \in \mathcal{J}_{7}$, then

$$
F_{1}^{1}(D)<s_{0}^{i n}<F_{0}\left(D, s_{1}^{i n}\right)<F_{1}^{2}(D)<F_{2}^{2}(D)-s_{1}^{i n},
$$

as $s_{1}^{i n}<M_{1}^{2}\left(D_{1}\right)$. From Table $5, \mathrm{SS}_{1}$ and $\mathrm{SS}_{2}^{2}$ do not exist, and $\mathrm{SS}_{2}^{1}$ exists and is LES, and $\mathrm{SS}_{3}^{1}$ exists and is unstable. If $\left(D, s_{0}^{i n}, s_{1}^{i n}\right) \in \mathcal{J}_{8}$, then

$$
s_{0}^{i n}<F_{1}^{1}(D)<F_{0}\left(D, s_{1}^{i n}\right)<F_{1}^{2}(D)<F_{2}^{2}(D)-s_{1}^{i n} .
$$

484 From Table $5, \mathrm{SS}_{1}, \mathrm{SS}_{2}^{1}$ and $\mathrm{SS}_{2}^{2}$ do not exist and $\mathrm{SS}_{3}^{1}$ exists and is LES.

Assume that $M_{1}^{2}\left(D_{1}\right)<s_{1}^{\text {in }}$. Using Table $5, \mathrm{SS}_{3}^{1}$ and $\mathrm{SS}_{3}^{2}$ exist. In this case, we have $F_{1}^{1}(D)>$ $F_{2}^{1}(D)-s_{1}^{i n}$ and $F_{1}^{2}(D)>F_{2}^{2}(D)-s_{1}^{i n}$. If $\left(D, s_{0}^{i n}, s_{1}^{i n}\right) \in \mathcal{J}_{9}$, then

$$
s_{0}^{i n}<F_{1}^{1}(D)<F_{1}^{2}(D)<F_{0}\left(D, s_{1}^{i n}\right) .
$$

From Table $5, \mathrm{SS}_{1}, \mathrm{SS}_{2}^{1}$ and $\mathrm{SS}_{2}^{2}$ do not exist, $\mathrm{SS}_{0}$ and $\mathrm{SS}_{3}^{1}$ are $\operatorname{LES}$ and $\mathrm{SS}_{3}^{2}$ is unstable. If $\left(D, s_{0}^{\text {in }}, s_{1}^{\text {in }}\right) \in$ $\mathcal{J}_{10}$, then

$$
F_{1}^{1}(D)<s_{0}^{i n}<F_{1}^{2}(D)<F_{0}\left(D, s_{1}^{i n}\right) .
$$

From Table 5, $\mathrm{SS}_{1}$ and $\mathrm{SS}_{2}^{2}$ do not exist, $\mathrm{SS}_{0}$ and $\mathrm{SS}_{2}^{1}$ are LES, and $\mathrm{SS}_{3}^{1}$ and $\mathrm{SS}_{3}^{2}$ are unstable. If $\left(D, s_{0}^{\text {in }}, s_{1}^{\text {in }}\right) \in \mathcal{J}_{11}$, then

$$
F_{1}^{1}(D)<F_{1}^{2}(D)<s_{0}^{i n}<F_{0}\left(D, s_{1}^{i n}\right) .
$$

From Table 5, $\mathrm{SS}_{1}$ does not exist, $\mathrm{SS}_{0}$ and $\mathrm{SS}_{2}^{1}$ are LES, and $\mathrm{SS}_{2}^{2}, \mathrm{SS}_{3}^{1}$ and $\mathrm{SS}_{3}^{2}$ are unstable. If $\left(D, s_{0}^{i n}, s_{1}^{i n}\right) \in \mathcal{J}_{12}$, then

$$
F_{2}^{1}(D)-s_{1}^{i n}<F_{1}^{1}(D)<F_{1}^{2}(D)<F_{0}\left(D, s_{1}^{i n}\right)<s_{0}^{i n} .
$$


Proof of Proposition 4.2. Let $D \in \bar{I}_{j}$. We have $s_{1 j}^{i n *}=M_{1}^{j}\left(D_{1}\right) \geq 0, j=1,2$ such that $s_{11}^{\text {in* }}<s_{12}^{\text {in* }}$ because $M_{1}^{1}\left(D_{1}\right) \leq M_{1}^{2}\left(D_{1}\right)$, for all $D \in \bar{I}_{2}$. For all $D \in I_{1}$, one has

$$
F_{0}\left(D, s_{11}^{i n *}\right)=M_{0}\left(D_{0}, M_{1}^{1}\left(D_{1}\right)\right)=F_{1}^{1}(D)<+\infty=F_{0}\left(D, \bar{s}_{1}^{i n}\right) .
$$

As the function $F_{0}(D, \cdot)$ is increasing, we obtain $s_{11}^{i n *}<\bar{s}_{1}^{i n}$. For all $D \in I_{1}$, we have

$$
F_{1}^{1}(D)=F_{1}^{1}(D)+M_{1}^{1}\left(D_{1}\right)-s_{11}^{i n *}=F_{2}^{1}(D)-s_{11}^{i n *},
$$

that is, the curves $\gamma_{0}, \gamma_{1}^{1}$ and $\gamma_{2}^{1}$ intersect at $s_{11}^{i n *}$. For all $s_{1}^{i n} \in\left[0, s_{11}^{i n *}\right)$, one has $M_{1}^{j}\left(D_{1}\right)>s_{1}^{i n}$ and therefore

$$
M_{0}\left(D_{0}, s_{1}^{i n}\right)<M_{0}\left(D_{0}, M_{1}^{1}\left(D_{1}\right)\right)<M_{0}\left(D_{0}, M_{1}^{1}\left(D_{1}\right)\right)+M_{1}^{1}\left(D_{1}\right)-s_{1}^{i n},
$$

that is, (4.2) holds. Inversely, for all $s_{1}^{i n} \in\left(s_{11}^{i n *}, \bar{s}_{1}^{i n}\right)$, (4.3) holds. The second assertion is proved in a similar manner.

Proof of Proposition 4.3. Let $s_{1}^{i n} \leq s_{1}^{\max }$. The function $D \mapsto M_{1}^{2}\left(\alpha_{1} D+a_{1}\right)-s_{1}^{i n}$ is nonnegative for all $D \in \bar{I}_{2}$ since it is decreasing from $M_{1}^{2}\left(a_{1}\right)-s_{1}^{\text {in }}$ for $D=0$ to $s_{1}^{\max }-s_{1}^{\text {in }} \geq 0$ for $D=\bar{D}_{1}$. Thus, for all $D \in I_{2}$,

$$
F_{1}^{2}(D)=M_{0}\left(D_{0}, M_{1}^{2}\left(D_{1}\right)\right) \leq M_{0}\left(D_{0}, M_{1}^{2}\left(D_{1}\right)\right)+M_{1}^{2}\left(D_{1}\right)-s_{1}^{i n}=F_{2}^{2}(D)-s_{1}^{i n} .
$$

Since $M_{0}$ is increasing with respect to the second variable (see formula (A.9)), we have for all $D \in I_{2}$,

$$
F_{0}\left(D, s_{1}^{i n}\right)=M_{0}\left(D_{0}, s_{1}^{i n}\right) \leq M_{0}\left(D_{0}, M_{1}^{2}\left(D_{1}\right)\right)=F_{1}^{2}(D) .
$$

Since the function $D \mapsto M_{1}^{1}\left(\alpha_{1} D+a_{1}\right)-s_{1}^{\text {in }}$ is increasing from $M_{1}^{1}\left(a_{1}\right)-s_{1}^{\text {in }}$ for $D=0$ to $s_{1}^{\text {max }}-s_{1}^{\text {in }}$ for $D=\bar{D}_{1}$, there exists a solution $D_{1}^{*} \in \bar{I}_{1}$ of equation $M_{1}^{1}\left(\alpha_{1} D+a_{1}\right)=s_{1}^{\text {in }}$ if and only if (4.6) holds. If such $D_{1}^{*}$ exists then it is unique. If $D_{1}^{*} \in I_{1}$, the function $F_{i}^{1}$ is defined for $D=D_{1}^{*}$ where

$$
F_{2}^{1}\left(D_{1}^{*}\right)-s_{1}^{i n}=M_{0}\left(\alpha_{0} D_{1}^{*}+a_{0}, M_{1}^{1}\left(\alpha_{1} D_{1}^{*}+a_{1}\right)\right)=F_{1}^{1}\left(D_{1}^{*}\right)=M_{0}\left(\alpha_{0} D_{1}^{*}+a_{0}, s_{1}^{i n}\right)=F_{0}\left(D_{1}^{*}, s_{1}^{i n}\right) .
$$

Since $M_{1}^{1}\left(D_{1}\right)<s_{1}^{i n}$ if $D<D_{1}^{*}$, one has

$$
F_{2}^{1}(D)-s_{1}^{i n}=M_{0}\left(D_{0}, M_{1}^{1}\left(D_{1}\right)\right)+M_{1}^{1}\left(D_{1}\right)-s_{1}^{i n}<M_{0}\left(D_{0}, M_{1}^{1}\left(D_{1}\right)\right)=F_{1}^{1}(D)<M_{0}\left(D_{0}, s_{1}^{i n}\right),
$$

that is, (4.3) holds. Inversely, if $D>D_{1}^{*}$, then (4.2) holds. If $D_{1}^{*} \in \bar{I}_{1} \backslash I_{1}$, that is, $D_{1}^{*} \in\left[\hat{D}_{1}, \bar{D}_{1}\right]$, then $M_{1}^{1}\left(\alpha_{1} D+a_{1}\right)<s_{1}^{\text {in }}$ for all $D \in\left[0, \hat{D}_{1}\right)$. Therefore, (4.3) holds where $\bar{D}_{0}\left(s_{1}^{i n}\right)<\hat{D}_{1}$. If $D_{1}^{*}$ does not exist, then $M_{1}^{1}\left(\alpha_{1} D+a_{1}\right)>s_{1}^{i n}$ for all $D \in I_{1}$ and consequently (4.2) holds.

Proof of Proposition 4.5. From Table 6 , the surface $\gamma_{0}$ is defined by $s_{0}^{i n}=F_{0}\left(D, s_{1}^{i n}\right)$. Using Tables 4 and 5 and Proposition 3.1, we can see that $\mathrm{SS}_{0}$ and $\mathrm{SS}_{1}$ coalesce and are nonhyperbolic steady states on the surface $\gamma_{0}$. Using Table 5 , if $s_{1}^{\text {in }}<M_{1}^{1}\left(D_{1}\right)$, we have a transition from $\mathcal{J}_{1}$ to $\mathcal{J}_{2}$ where $\mathrm{SS}_{0}$ becomes unstable and $\mathrm{SS}_{1}$ emerges stable in the positive octant $\mathbb{R}_{+}^{4}$. All other cases are left to the reader since they can be treated similarly.

Appendix C. The particular case for growth functions (C.1). The Hypotheses 2.1 to 2.5 are satisfied by the following growth rates of Monod-type with hydrogen inhibition and of Haldane-type, respectively,

$$
\mu_{0}\left(s_{0}, s_{1}\right)=\frac{m_{0} s_{0}}{K_{0}+s_{0}} \frac{1}{1+s_{1} / K_{i}}, \quad \mu_{1}\left(s_{1}\right)=\frac{m_{1} s_{1}}{K_{1}+s_{1}+s_{1}^{2} / K_{I}},
$$

where $m_{j}$ and $K_{j}, j=0,1$, denote the maximum growth rates (units are $1 /$ d) and the Michaelis-Menten constants with units of concentration $\left(\mathrm{kg} \mathrm{COD} / \mathrm{m}^{3}\right) ; K_{i}$ and $K_{I}$ represent the inhibition factor due to $s_{1}$ for the growth of the species $x_{0}$ and $x_{1}$, respectively, with units of concentration. The following result determines the maximal number of solutions of the equation $\Phi_{2}(D)=0$ in the particular case of growth functions (C.1) when $\alpha_{0}>0$ and $\alpha_{1}>0$. 

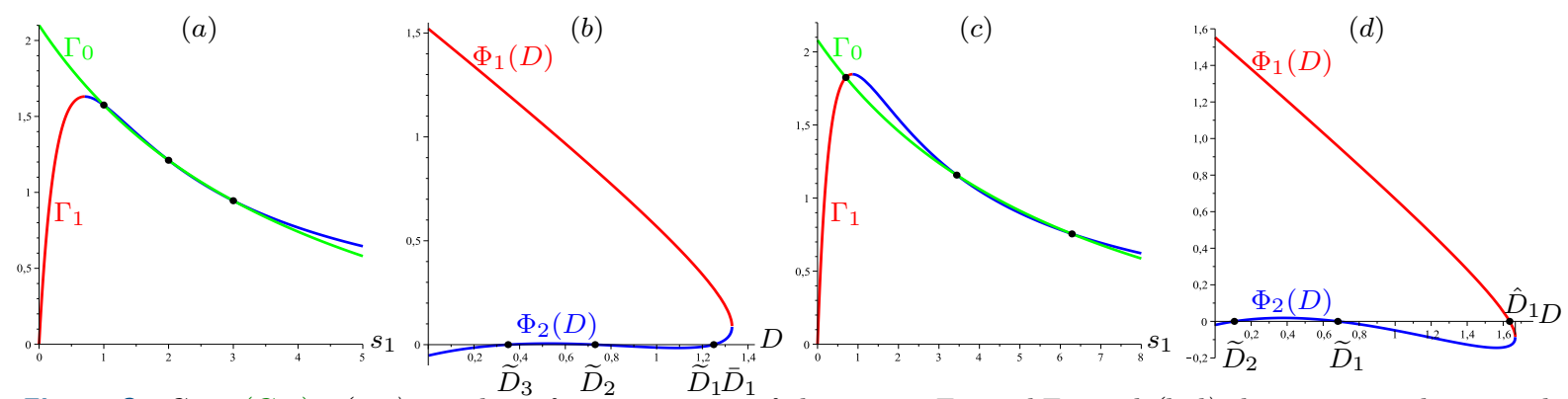

Figure 8. Case (C.3): (a,c) number of intersections of the curves $\Gamma_{0}$ and $\Gamma_{1}$ and $(b, d)$ the corresponding number of solutions of equation $\Phi_{j}(D)=0$. (a-b) In case 1 of $(4.1)$, the equation $\Phi_{2}(D)=0$ has three solutions on $\left[0, \bar{D}_{1}\right] .(c-d)$ In case 2 of (4.1), the equation $\Phi_{2}(D)=0$ has two solutions on $\left[0, \bar{D}_{1}\right]$.
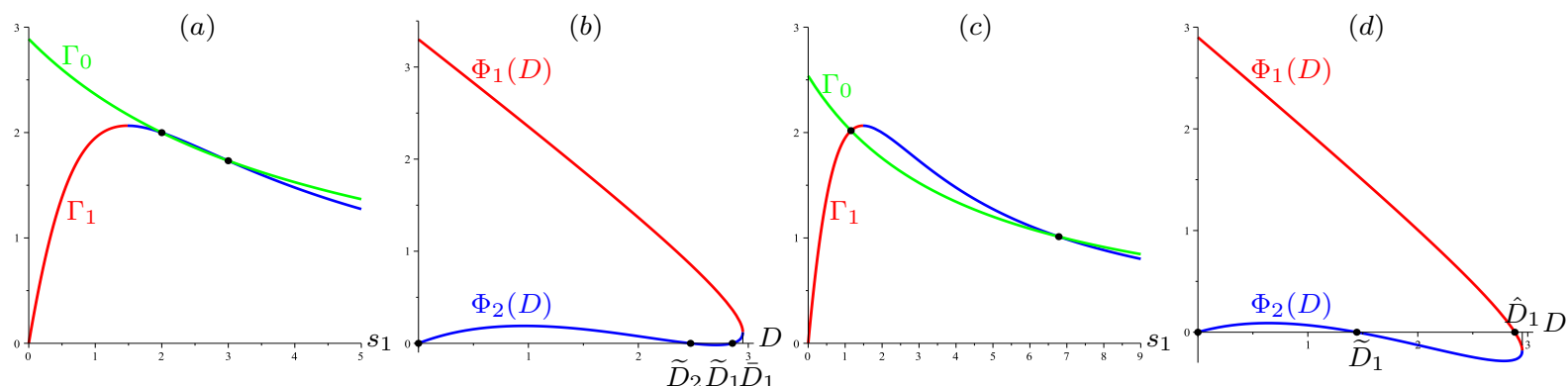

Figure 9. Case (C.4), in particular without decay: (a,c) number of intersections of the curves $\Gamma_{0}$ and $\Gamma_{1}$ of the functions $\widetilde{\mu}_{0}$ and $\mu_{1}$, respectively, and $(b, d)$ the corresponding number of solutions of equation $\Phi_{j}(D)=0$. (a-b) In case 1 of $(4.1)$, the equation $\Phi_{2}(D)=0$ has two solutions on $\left[0, \bar{D}_{1}\right]$. (c-d) In case 2 of $(4.1)$, the equation $\Phi_{2}(D)=0$ has one solution on $\left[0, \bar{D}_{1}\right]$.

Proposition C.1. Let $\alpha_{0}>0$ and $\alpha_{1}>0$. The equations $\Phi_{1}(D)=0$ and $\Phi_{2}(D)=0$ are equivalent

$$
\mu_{1}(y)=\widetilde{\mu}_{0}(y):=\frac{\alpha_{1}}{\alpha_{0}} \bar{\mu}_{0}(y)+a_{1}-\frac{\alpha_{1}}{\alpha_{0}} a_{0}, \text { with } y=\bar{\mu}_{0}^{-1}\left(D_{0}\right) .
$$

When the growth functions $\mu_{0}$ and $\mu_{1}$ are of type (C.1), the equation $\Phi_{2}(D)=0$ has at most three solutions in the case 1 of (4.1) when

$$
a_{1} / \alpha_{1} \neq a_{0} / \alpha_{0},
$$

and at most two solutions in the case 2 of (4.1) (see Figure 8(b-d)). It has at most two solutions in

$$
a_{1} / \alpha_{1}=a_{0} / \alpha_{0},
$$

and at most one solution in the case 2 of (4.1), (see Figure 9(b-d)).

Proof. From definition of the function $\Phi_{j}$ in Table 9 and Hypothesis 2.5, we have, for $j=1,2$,

$$
\Phi_{j}(D)=0 \Longleftrightarrow M_{1}^{j}\left(D_{1}\right)=\bar{\mu}_{0}^{-1}\left(D_{0}\right) \Longleftrightarrow \mu_{1}\left(\bar{\mu}_{0}^{-1}\left(D_{0}\right)\right)=D_{1} .
$$

Let $y=\bar{\mu}_{0}^{-1}\left(D_{0}\right)$. Thus, $D_{0}=\bar{\mu}_{0}(y)$ and $D_{1}=\mu_{1}(y)$. From definition (1.3) of $D_{i}, i=0,1$, we have $D_{1}=\alpha_{1}\left(D_{0}-a_{0}\right) / \alpha_{0}+a_{1}$. Consequently, equation (C.2) holds. When the growth functions $\mu_{0}$ and $\mu_{1}$ are of type (C.1), we obtain

$$
\frac{m_{1} y}{K_{1}+y+y^{2} / K_{I}}=\frac{K_{i} m_{0} \alpha_{1} / \alpha_{0}+\left(K_{i}+y\right)\left(a_{1}-a_{0} \alpha_{1} / \alpha_{0}\right)}{K_{i}+y} .
$$

When condition (C.3) holds, we obtain an algebraic equation of degree three in $y$ and consequently the equation (C.2) has at most three solutions. Hence, if case 1 of (4.1) holds, that is, the equation $\Phi_{1}(D)=0$ has no solution, then the equation $\Phi_{2}(D)=0$ has at most three solutions. However, if case 2 of (4.1) holds, that is, the equation $\Phi_{1}(D)=0$ has one solution, then the equation $\Phi_{2}(D)=0$ has at most two solutions. When condition (C.4) holds, we obtain an algebraic equation of degree two in $y$. Thus, the rest of the results follows similarly. 
When the growth functions are given by (C.1), we succeeded in finding a set of parameters such that we show the maximum number of intersections of the curves $\Gamma_{0}$ and $\Gamma_{1}$ and the corresponding number of solutions of the equation $\Phi_{j}(D)=0$ (see Figures 8 and 9).

Appendix D. Tables. In this section, we give some tables used in the paper. Tables 10 and 11 describe the intersections of the surfaces of $\Gamma$ with a two-dimensional operating plane where $D$ or $s_{1}^{\text {in }}$ is constant, respectively. Table 12 presents the auxiliary functions defined in Table 3 in the particular case of the Monod-type with hydrogen inhibition and of the Haldane-type given by (C.1). Table 13 provides the biological parameter values used in all the figures.

Table 10

Intersections of the surfaces of $\Gamma$ with a $\left(s_{1}^{i n}, s_{0}^{i n}\right)$ plane where $D$ is constant.

\begin{tabular}{lll}
$\Gamma$ & Condition & $\Gamma \cap\{D=$ constant $\}$ \\
\hline \multirow{2}{*}{$\gamma_{0}$} & $D<\bar{D}_{0}(0)$ & Curve of the function $s_{0}^{i n}=F_{0}\left(D, s_{1}^{i n}\right)$ \\
& $D \geq \bar{D}_{0}(0)$ & Empty \\
\hline \multirow{2}{*}{$\gamma_{1}^{j}$} & $D \in I_{j}$ & Horizontal line $s_{0}^{i n}=F_{1}^{j}(D)$ \\
& $D \notin I_{j}$ & Empty \\
\multirow{2}{*}{$\gamma_{2}^{j}$} & $D \in I_{j}$ & Oblique line $s_{0}^{i n}=F_{2}^{j}(D)-s_{1}^{i n}$ \\
& $D \notin I_{j}$ & Empty \\
\multirow{2}{*}{$\gamma_{3}^{j}$} & $D \in \bar{I}_{j}$ & Vertical line $s_{1}^{i n}=s_{1 j}^{i n *}=M_{1}^{j}\left(D_{1}\right)$ \\
& $D \notin \bar{I}_{j}$ & Empty \\
\multirow{2}{*}{$\gamma_{4}$} & $D=\bar{D}_{1}$ & Whole plane $\left(s_{1}^{i n}, s_{0}^{i n}\right)$ \\
& $D \neq \bar{D}_{1}$ & Empty
\end{tabular}

Table 11

Intersections of the surfaces of $\Gamma$ with a $\left(D, s_{0}^{\text {in }}\right)$ plane where $s_{1}^{\text {in }}$ is constant.

\begin{tabular}{lll}
$\Gamma$ & Condition & $\Gamma \cap\left\{s_{1}^{i n}=\right.$ constant $\}$ \\
\hline \multirow{2}{*}{$\gamma_{0}$} & $D<\bar{D}_{0}\left(s_{1}^{i n}\right)$ & Curve of the function $s_{0}^{i n}=F_{0}\left(D, s_{1}^{i n}\right)$ \\
& $D \geq \bar{D}_{0}\left(s_{1}^{i n}\right)$ & Empty \\
\hline \multirow{2}{*}{$\gamma_{1}^{j}$} & $D \in I_{j}$ & Curve of the function $s_{0}^{\text {in }}=F_{1}^{j}(D)$ \\
& $D \notin I_{j}$ & Empty \\
\hline \multirow{2}{*}{$\gamma_{2}^{j}$} & $D \in I_{j}$ and $s_{1}^{\text {in }}<F_{2}^{j}(D)$ & Curve of the function $s_{0}^{\text {in }}=F_{2}^{j}(D)-s_{1}^{\text {in }}$ \\
& $D \notin I_{j}$ or $s_{1}^{\text {in }}>F_{2}^{j}(D)$ & Empty \\
\hline \multirow{2}{*}{$\gamma_{3}^{1}$} & $s_{1}^{\text {in }} \in\left(M_{1}^{1}\left(a_{1}\right), s_{1}^{\text {max }}\right]$ & Vertical line $D=D_{1}^{*}$ \\
& $s_{1}^{\text {in }} \notin\left(M_{1}^{1}\left(a_{1}\right), s_{1}^{\text {max }}\right]$ & Empty \\
\hline \multirow{2}{*}{$\gamma_{3}^{2}$} & $s_{1}^{\text {in }} \in\left[s_{1}^{\text {max }}, M_{1}^{2}\left(a_{1}\right)\right)$ & Vertical line $D=D_{2}^{*}$ \\
& $s_{1}^{\text {in }} \notin\left[s_{1}^{\max }, M_{1}^{2}\left(a_{1}\right)\right)$ & Empty \\
\hline$\gamma_{4}$ & $s_{0}^{\text {in }} \geq \max \left(0, s_{1}^{\text {max }}-s_{1}^{\text {in }}\right)$ & Vertical line $D=D_{1}$
\end{tabular}

Table 12

Auxiliary functions with the specific growth functions (C.1) where $I_{j}, j=1,2$ are defined by (A.2), (A.5), and (A.6).

\begin{tabular}{ll} 
Auxiliary function & Definition domain \\
\hline$M_{0}\left(y, s_{1}\right)=\frac{K_{0} y\left(1+s_{1} / K_{i}\right)}{m_{0}-y\left(1+s_{1} / K_{i}\right)}$ & $0 \leq y<\frac{m_{0}}{1+s_{1} / K_{i}}$ \\
$M_{1}^{j}(y)=\frac{\left(m_{1}-y\right) K_{I} \pm \sqrt{\left(K_{I}\left(m_{1}-y\right)\right)^{2}-4 K_{1} K_{I} y^{2}}}{2 y}$ & $0<y<\frac{m_{1}}{1+2 \sqrt{K_{1} / K_{I}}}$ \\
$F_{0}\left(D, s_{1}^{i n}\right)=\frac{K_{0}\left(\alpha_{0} D+a_{0}\right)\left(1+s_{1}^{i n} / K_{i}\right)}{m_{0}-\left(\alpha_{0} D+a_{0}\right)\left(1+s_{1}^{i n} / K_{i}\right)}$ & $0 \leq \alpha_{0} D+a_{0}<\frac{m_{0}}{1+s_{1}^{i n} / K_{i}}$ \\
$F_{1}^{j}(D)=\frac{K_{0}\left(\alpha_{0} D+a_{0}\right)\left(1+M_{1}^{j}\left(\alpha_{1} D+a_{1}\right) / K_{i}\right)}{m_{0}-\left(\alpha_{0} D+a_{0}\right)\left(1+M_{1}^{j}\left(\alpha_{1} D+a_{1}\right) / K_{i}\right)}$ & $D \in I_{j}$ \\
$F_{2}^{j}(D)=M_{1}^{j}\left(\alpha_{1} D+a_{1}\right)+F_{1}^{j}(D)$ & $D \in I_{j}$
\end{tabular}

[1] N. Abdellatif, R. Fekih-Salem, and T. Sari, Competition for a single resource and coexistence of several species in the chemostat, Math. Biosci. Eng., 13 (2016), pp. 631-652, https://doi.org/10.3934/mbe.2016012. Discrete \& Continuous Dyn. Syst. - B, 25 (2020), pp. 2093-2120, https://doi.org/10.3934/dcdsb.2019203. 
Table 13

The biological parameter values used for system (2.1) with the specific growth functions (C.1).

\begin{tabular}{lllllllllll} 
Parameter & $m_{0}$ & $K_{0}$ & $K_{i}$ & $m_{1}$ & $K_{1}$ & $K_{I}$ & $\alpha_{0}$ & $a_{0}$ & $\alpha_{1}$ & $a_{1}$ \\
\hline Figures 1, 3, and 5, Figure 7(a) & 3.5 & 1.5 & 5 & 4 & 0.5 & 0.95 & 0.9 & 1.2 & 0.8 & 0.7 \\
Figures 2, 4, and 6 & 3.5 & 1.5 & 5 & 3.5 & 0.5 & 0.95 & 0.95 & 1.4 & 0.5 & 0.5 \\
Figure 7(b) & 4 & 1.5 & 1.2 & 5.32 & 0.5 & 0.95 & 1 & 1 & 0.8 & 1.18 \\
Figure 7(c), Figure 8(a,b) & 3.3 & 1 & 4.5 & 3.94 & 0.5 & 1 & 0.8 & 1.7 & 0.7 & 0.7 \\
Figure 8(c,d) & 3.2 & 1 & 7 & 3.98 & 0.5 & 1.5 & 0.8 & 1.6 & 0.7 & 0.68 \\
Figure 9(a-b) & 3.3 & 1 & 4.5 & 8.21 & 2.21 & 1 & 0.8 & 0 & 0.7 & 0 \\
Figure 9(c-d) & 2.9 & 1 & 4.5 & 8.21 & 2.21 & 1 & 0.8 & 0 & 0.7 & 0
\end{tabular}

[3] D. Batstone, J. Keller, I. Angelidaki, S. Kalyhuzhnyi, S. Pavlosthathis, A. Rozzi, W. Sanders, H. Siegrist, and V. Vavilin, The IWA Anaerobic Digestion Model No 1 (ADM1), Water Sci Technol., 45 (2002), pp. 66-73, https://doi.org/ 10.2166 /wst.2002.0292.

[4] B. Benyahia and T. Sari, Effect of a new variable integration on steady states of a two-step anaerobic digestion model, Math. Biosci. Eng., 17 (2020), pp. 5504-5533.

[5] B. Benyahia, T. Sari, B. Cherki, and J. Harmand, Bifurcation and stability analysis of a two step model for monitoring anaerobic digestion processes, J. Proc. Control, 22 (2012), pp. 1008-1019, https://doi.org/10.1016/j.jprocont. 2012.04.012

[6] B. Benyahia, T. Sari, B. Cherki, and J. Harmand, Anaerobic membrane bioreactor modeling in the presence of Soluble Microbial Products (SMP) - the Anaerobic Model AM2b, Chem. Eng. J., 228 (2013), pp. 1011-1022, https: //doi.org/10.1016/j.cej.2013.05.073.

[7] O. Bernard, Z. Hadj-Sadok, D. Dochain, A. Genovesi, and J.-P. Steyer, Dynamical model development and parameter identification for an anaerobic wastewater treatment process, Biotechnol. Bioeng., 75 (2001), pp. 424-438, https://doi.org/10.1002/bit.10036.

[8] M. Boer, B. Kooi, and S. Kooijman, Food chain dynamics in the chemostat, Math. Biosci., 150 (1998), pp. 43-62, https://doi.org/10.1016/S0025-5564(98)00010-8.

[9] A. Bornhöft, R. Hanke-Rauschenbach, and K. Sundmacher, Steady-state analysis of the anaerobic digestion model No. 1 (ADM1), Nonlinear Dyn., 73 (2013), pp. 535-549, https://doi.org/10.1007/s11071-013-0807-x.

[10] A. Burchard, Substrate degradation by a mutualistic association of two species in the chemostat, J. Math. Biol., 32 (1994), pp. 465-489, https://doi.org/10.1007/BF00160169.

[11] Y. Daoud, N. Abdellatif, T. Sari, and J. Harmand, Steady state analysis of a syntrophic model: The effect of a new input substrate concentration, Math. Model. Nat. Phenom., 13 (2018), pp. 1-22, https://doi.org/10.1051/mmnp/ 2018037.

[12] M. Dellal and B. Bar, Global analysis of a model of competition in the chemostat with internal inhibitor, Discrete \& Continuous Dyn. Syst. - B, (2020), https://doi.org/10.3934/dcdsb.2020156.

[13] M. Dellal, M. Lakrib, and T. Sari, The operating diagram of a model of two competitors in a chemostat with an external inhibitor, Math. Biosci., 302 (2018), pp. 27-45, https://doi.org/10.1016/j.mbs.2018.05.004.

[14] S. Di and A. Yang, Analysis of productivity and stability of synthetic microbial communities, J. R. Soc. Interface, 16 (2019), pp. 1-19, https://doi.org/10.1098/rsif.2018.0859.

[15] A. Donoso-Bravo, J. Mailier, C. Martin, J. Rodríguez, C. A. Aceves-Lara, and A. Vande Wouwer, Model selection, identification and validation in anaerobic digestion: A review, Water Research, 45 (2011), pp. 5347-5364, https://doi.org/10.1016/j.watres.2011.08.059.

[16] M. El-Hajji, F. Mazenc, and J. Harmand, A mathematical study of a syntrophic relationship of a model of anaerobic digestion process, Math. Biosci. Eng., 7 (2010), pp. 641-656, https://doi.org/10.3934/mbe.2010.7.641.

[17] R. Fekih-Salem, N. Abdellatif, T. Sari, and J. Harmand, Analyse mathématique d'un modèle de digestion anaérobie à trois étapes, ARIMA J., 17 (2014), pp. 53-71, http://arima.inria.fr/017/017003.html.

[18] R. Fekih-Salem, N. Abdellatif, and A. Yahmadi, Effect of inhibition on a syntrophic relationship model in the anaerobic digestion process, in Proceedings of the 8th conference on Trends in Applied Mathematics in Tunisia, Algeria, Morocco, 2017, pp. 391-396, https://indico.math.cnrs.fr/event/1335.

[19] R. Fekih-Salem, C. Lobry, and T. Sari, A density-dependent model of competition for one resource in the chemostat, Math. Biosci., 286 (2017), pp. 104-122, https://doi.org/10.1137/18M1171801, http://www.sciencedirect.com/ science/article/pii/S0025556417300792.

[20] F. Gantmacher, Application of the theory of matrices, Interscience Publishers, INC. New York, 2004.

[21] A. Ghouali, T. Sari, and J. Harmand, Maximizing biogas production from the anaerobic digestion, J. Process Control, 36 (2015), pp. 79-88, https://doi.org/10.1016/j.jprocont.2015.09.007.

[22] G. Giovannini, M. Sbarciog, J.-P. Steyer, R. Chamy, and A. Vande Wouwer, On the derivation of a simple dynamic model of anaerobic digestion including the evolution of hydrogen, Water Research, 134 (2018), pp. 209-225, https://doi.org/10.1016/j.watres.2018.01.036.

[23] J. Harmand, C. Lobry, A. Rapaport, and T. Sari, The Chemostat: Mathematical Theory of Microorganism Cultures, vol. 1, Chemical Eng. Ser., Chemostat Bioprocesses Set, Wiley, New York, 2017, https://doi.org/10.1002/ 9781119437215 .

[24] E. Harvey, J. Heys, and T. Gedeon, Quantifying the effects of the division of labor in metabolic pathways, J. Theor. Biol., 360 (2014), pp. 222-242, https://doi.org/10.1016/j.jtbi.2014.07.011. 
[25] Z. Khedim, B. Benyahia, B. Cherki, T. Sari, and J. Harmand, Effect of control parameters on biogas production during the anaerobic digestion of protein-rich substrates, Appl. Math. Model., 61 (2018), pp. 351-376, https://doi.org/ 10.1016/j.apm.2018.04.020.

[26] R. Kreikenbohm and E. Bohl, A mathematical model of syntrophic cocultures in the chemostat, FEMS Microbiol. Ecol., 38 (1986), pp. 131-140, https://doi.org/10.1111/j.1574-6968.1986.tb01722.x.

[27] J. Mailier, M. Remy, and A. Vande Wouwer, Stoichiometric identification with maximum likelihood principal component analysis, J. Math. Biol., 67 (2013), pp. 739-765, https://doi.org/10.1007/s00285-012-0559-0.

[28] F. Mairet, O. Bernard, E. Cameron, M. Ras, L. Lardon, J.-P. Steyer, and B. Chachuat, Three-reaction model for the anaerobic digestion of microalgae, Biotechnol. Bioeng., 109 (2012), pp. 415-425, https://doi.org/10.1002/bit. 23350.

[29] S. Marsili-Libelli and S. Beni, Shock load modelling in the anaerobic digestion process, Ecol. Model., 84 (1996), pp. 215-232, https://doi.org/10.1016/0304-3800(94)00125-1.

[30] C. Martinez, A. Ávila, F. Mairet, L. Meier, and D. Jeison, Modeling and analysis of an absorption column connected to a microalgae culture, SIAM J. Appl. Math., 80 (2020), pp. 772-791, https://doi.org/10.1137/18M1225641.

[31] T. Meadows, M. Weedermann, and G. Wolkowicz, Global analysis of a simplified model of anaerobic digestion and a new result for the chemostat, SIAM J. Appl. Math., 79 (2019), pp. 668-689, https://doi.org/10.1137/18M1198788.

[32] T. Mtar, R. Fekih-Salem, and T. Sari, Interspecific density-dependent model of predator-prey relationship in the chemostat, Int. J. Biomath., (2020), https://hal.archives-ouvertes.fr/hal-02445761.

[33] B.-J. Ni, G.-P. Sheng, and H.-Q. Yu, Model-based characterization of endogenous maintenance, cell death and predation processes of activated sludge in sequencing batch reactors, Chem. Eng. Sci., 66 (2011), pp. 747-754, https: //doi.org/10.1016/j.ces.2010.11.033.

[34] S. Nouaoura, N. Abdellatif, R. Fekih-Salem, and T. Sari, Mathematical analysis of a three-tiered model of anaerobic digestion. hal-02540350, (2020), https://hal.archives-ouvertes.fr/hal-02540350.

[35] S. Nouaoura, R. Fekih-Salem, N. Abdellatif, and T. Sari, Mathematical analysis of a three-tiered food-web in the chemostat, To appear in Discrete \& Continuous Dyn. Syst. - B, (2020), https://hal.archives-ouvertes.fr/hal-02878246.

[36] S. Pavlou, Computing operating diagrams of bioreactors, J. Biotechnol., 71 (1999), pp. 7-16, https://doi.org/10. 1016/S0168-1656(99)00011-5.

[37] P. J. Reilly, Stability of commensalistic systems, Biotechnol. Bioeng., 16 (1974), pp. 1373-1392, https://doi.org/10. 1002/bit.260161006.

[38] T. Sari and B. Benyahia, The operating diagram for a two-step anaerobic digestion model. hal-02557464, (2020), https://hal.archives-ouvertes.fr/hal-02557464.

[39] T. Sari, M. E. Hajji, and J. Harmand, The mathematical analysis of a syntrophic relationship between two microbial species in a chemostat, Math. Biosci. Eng., 9 (2012), pp. 627-645, https://doi.org/10.3934/mbe.2012.9.627.

[40] T. Sari and J. Harmand, A model of a syntrophic relationship between two microbial species in a chemostat including maintenance, Math. Biosci., 275 (2016), pp. 1-9, https://doi.org/10.1016/j.mbs.2016.02.008.

[41] T. Sari and M. Wade, Generalised approach to modelling a three-tiered microbial food-web, Math. Biosci., 291 (2017), pp. 21-37, https://doi.org/10.1016/j.mbs.2017.07.005.

[42] M. Sbarciog, M. Loccufier, and E. Noldus, Determination of appropriate operating strategies for anaerobic digestion systems, Biochem. Eng. J., 51 (2010), pp. 180-188, https://doi.org/10.1016/j.bej.2010.06.016.

[43] M. Sbarciog, M. Loccufier, and A. Vande Wouwer, An optimizing start-up strategy for a bio-methanator, Bioprocess Biosyst Eng, 35 (2012), pp. 565-578, https://doi.org/10.1007/s00449-011-0629-5.

[44] M. Sbarciog, J. A. Moreno, and A. Vande Wouwer, A biogas-based switching control policy for anaerobic digestion systems, IFAC Proceedings, 45 (2012), pp. 603-608, https://doi.org/10.3182/20120710-4-SG-2026.00056.

[45] S. Shen, G. C. Premier, A. Guwy, and R. Dinsdale, Bifurcation and stability analysis of an anaerobic digestion model, Nonlinear Dynam., 48 (2007), pp. 391-408, https://doi.org/10.1007/s11071-006-9093-1.

[46] I. Simeonov and S. Diop, Stability analysis of some nonlinear anaerobic digestion models, Int. J. Bioautomation, 14 (2010), pp. 37-48.

[47] I. Simeonov and S. Stoyanov, Modelling and dynamic compensator control of the anaerobic digestion of organic wastes, Chem. Biochem. Eng. Q., 17 (2003), pp. 285-292.

[48] G. Stephanopoulos, The dynamics of commensalism, Math. Biosci., 23 (1981), pp. 2243-2255, https://doi.org/10. 1002/bit.260231008.

[49] E. Volcke, M. Sbarciog, E. Noldus, B. D. Baets, and M. Loccufier, Steady state multiplicity of two-step biological conversion systems with general kinetics, Math. Biosci., 228 (2010), pp. 160-170, https://doi.org/10.1016/j.mbs. 2010.09.004.

[50] M. Wade, Not just numbers: Mathematical modelling and its contribution to anaerobic digestion processes, Processes, 8 (2020), p. 888, https://doi.org/10.3390/pr8080888.

[51] M. Wade, R. Pattinson, N. Parker, and J. Dolfing, Emergent behaviour in a chlorophenol-mineralising three-tiered microbial 'food web', J. Theor. Biol., 389 (2016), pp. 171-186, https://doi.org/10.1016/j.jtbi.2015.10.032.

[52] M. Weedermann, G. Seo, and G. S. Wolkowicz, Mathematical model of anaerobic digestion in a chemostat: effects of syntrophy and inhibition, J. Biol. Dyn., 7 (2013), pp. 59-85, https://doi.org/10.1080/17513758.2012.755573.

[53] M. Weedermann, G. S. Wolkowicz, and J. Sasara, Optimal biogas production in a model for anaerobic digestion, Nonlinear Dyn, 81 (2015), pp. 1097-1112, https://doi.org/10.1007/s11071-015-2051-z.

[54] A. Xu, J. Dolfing, T. Curtis, G. Montague, and E. Martin, Maintenance affects the stability of a two-tiered microbial 'food chain'?, J. Theor. Biol., 276 (2011), pp. 35-41, https://doi.org/10.1016/j.jtbi.2011.01.026. 